\title{
The quench dynamics of a dissipative quantum system: a renormalization group study
}

\author{
O. Kashuba, D. M. Kennes, M. Pletyukhov, V. Meden, and H. Schoeller \\ Institut für Theorie der Statistischen Physik, RWTH Aachen University and \\ JARA-Fundamentals of Future Information Technology, 52056 Aachen, Germany
}

(Dated: October 29, 2018)

\begin{abstract}
We study dissipation in a small quantum system coupled to an environment held in thermodynamic equilibrium. The relaxation dynamics of a system subject to an abrupt quench in the parameters of the underlying Hamiltonian is investigated using two complementary renormalization group approaches. The methods are applied to the Ohmic spin-boson model close to the coherentto-incoherent transition. In particular, the role of non-Markovian memory for the relaxation before and after the quench of the spin-boson coupling and the Zeeman splitting of the up and down spin is investigated.
\end{abstract}

\section{INTRODUCTION}

Obtaining a detailed understanding of a dissipation in small quantum systems coupled to a reservoir held in thermodynamic equilibrium poses a formidable challenge. With the increasing accuracy of experimental techniques in diverse fields such as condensed matter physics, quantum optics, cold atomic gases, physical chemistry, and quantum information science, phenomenological approaches need to be complemented by a more microscopic view. One thus has to develop appropriate microscopic models for dissipation as well as the corresponding quantum many-body methods to solve those with sufficient precision. We here report on progress with respect to the second aspect. Focusing on the commonly investigated spin-boson model (SBM), $, 1,2$ we propose two complementary renormalization group (RG) based methods which allow controlled access to the relaxation dynamics of the Ohmic SBM close to its coherent-to-incoherent transition ${ }^{1,2}$ : the real-time RG (RTRG) (Ref. 3) and the functional RG (FRG) (Ref. 4). We study the relaxation dynamics under the SBM Hamiltonian out of an initially (at time $t=0$ ) prepared product state of spin-up and the boson vacuum together with the time evolution after a quantum quench where one of the parameters of the SBM is changed abruptly; we here exclusively consider temperature $T=0$.

The time dependence of the position of a classical damped harmonic oscillator constitutes a possible point of reference for the functional dependence of the spin expectation value $P$ on time $t$ in the SBM. An important intrinsic property of the SBM that is absent in the oscillator is the system's memory. Non-Markovian memory effects are a fundamental issue in dissipative quantum systems, in particular due to their relevance in quantum information theory. Several ways have been proposed to characterize and measure to which degree the dynamics of an open quantum system shows non-Markovian features, see e.g. Ref. 5 for a recent review. Here, we define nonMarkovian memory by those effects which result from the energy dependence of the effective kernel in Laplace space determining the time evolution of the local quantum system. Using RTRG, a generic analysis 6,7 has shown that
non-Markovian dynamics results in additional exponentially decaying terms for the time evolution, which have unexpected oscillation frequencies and decay rates compared to the leading Markovian terms, together with preexponential functions containing power laws and logarithmic functions in the long-time limit. These effects result from the nonanalytic energy dependence of the effective kernel, where the position of a branching point determines the exponential part, whereas the pre-exponential function follows from the scaling behavior around the branching point. In special cases, like e.g. quantum critical points or reservoirs with specific nonanalytic density of states, it may even happen that non-Markovian effects lead to a pure power law in the long-time limit without any exponentially decaying part, as it is e.g. the case for multi-channel Kondo models. 8

Non-Markovian effects have already been reported for the Ohmic SBM. Its Hamiltonian reads as

$$
\begin{aligned}
H_{\mathrm{tot}}= & \frac{\epsilon}{2} \sigma_{z}-\frac{\Delta}{2} \sigma_{x}+\sum_{k} \omega_{k} b_{k}^{\dagger} b_{k} \\
& -\sum_{k} \frac{\lambda_{k}}{2} \sigma_{z}\left(b_{k}^{\dagger}+b_{k}\right),
\end{aligned}
$$

where $\sigma_{\eta}, \eta=x, z$, are the Pauli matrices, and $b_{k}^{(\dagger)}$ are bosonic ladder operators. A spin- $\frac{1}{2}$ with Zeeman splitting $\epsilon$ and tunneling $\Delta \geq 0$ between the two states is coupled by $\lambda_{k}$ to a reservoir of bosonic modes with dispersion $\omega_{k}$. Of interest is the computation of the spin expectation value $P(t)=\left\langle\sigma_{z}\right\rangle(t)$ primarily considering the initial condition $\left\langle\sigma_{z}\right\rangle(0)=1$. $P(t)$ corresponds to the population imbalance of left and right double-well states when exploiting the analogy of the SBM to a particle in a double-well potential 1,2 The spin-boson coupling is characterized by a spectral density $J(\omega)=\sum_{k} \lambda_{k}^{2} \delta\left(\omega-\omega_{k}\right)$ containing the microscopic details of the model $\underline{1}$ We concentrate on the extensively studied ohmic case when the spectral density is linear up to a cutoff $D$, e.g. $J(\omega)=2 \alpha \omega e^{-\omega / \omega_{c}}$. In the special case without bias, $\epsilon=0$, the standard result quoted for the spin expectation value $P(t)$ is the one resulting from the noninteracting blip approximation (NIBA), where $P(t)$ decomposes into a coherent (pole) and incoherent (branch cut) contribution $P(t)=P_{\text {pole }}(t)+P_{\mathrm{bc}}(t)$. In the long-time limit, 
$T_{K} t \gg 1$, the NIBA predicts 1,2

$$
\begin{aligned}
P_{\text {pole }}(t) & =\theta(1-2 \alpha) \frac{1}{1-\alpha} e^{-\Gamma_{1}^{*} t} \cos (\Omega t) \\
P_{\mathrm{bc}}(t) & =\frac{1}{\Gamma(2 \alpha-1)} \frac{1}{\left(T_{K} t\right)^{2-2 \alpha}} .
\end{aligned}
$$

Here, $\Gamma_{1}^{*}=T_{K} \sin \frac{\pi \alpha}{2(1-\alpha)}$ and $\Omega=T_{K} \cos \frac{\pi \alpha}{2(1-\alpha)}$ are the characteristic scales determining the exponential time dynamics of the coherent part $P_{\text {pole }}(t)$, and $T_{K}=$ $\Delta\left(\Delta / \omega_{c}\right)^{\alpha /(1-\alpha)}$ is an effective tunneling rate. The incoherent part $P_{\mathrm{bc}}(t)$ is a term resulting purely from nonMarkovian dynamcis, whereas the coherent part $P_{\text {pole }}(t)$ is also obtained in Markov approximation but the precise values of $\Gamma_{1}^{*}, \Omega$, and the prefactor are influenced by non-Markovian contributions. For strong enough damping $\alpha>\frac{1}{2}$, the NIBA predicts correctly that the coherent term $P_{\text {pole }}(t)$ is absent. Although the results (2) and (3) were originally predicted for all values of $\alpha$, controlled calculations for small $\alpha$ and for $\alpha$ close to $\frac{1}{2}$ have shown that this result can not be correct for all $\alpha$. For small $\alpha$ it has been shown recently that $P_{\mathrm{bc}}(t)$ is also exponentially decaying with a rate $\Gamma_{2}^{*} \approx 2 \Gamma_{1}^{*}$ (Refs. 9 and 7 ) and the $O(\alpha)$ correction to the exponent of the algebraically decaying function is absent $\underline{\underline{7}}$. Especially the latter requires a controlled analysis close to branching points of the effective kernel, such that all powers $\sim\left[\alpha \ln \left(T_{K} t\right)\right]^{n}$ are systematically taken into account. So far, this has only been achieved by the RTRG method, where a controlled renormalization group study is possible for the weak-coupling regime of small $\alpha$.

For $\alpha$ close to the coherent-to-incoherent transition at $\alpha=\frac{1}{2}$, which is the regime of interest in this work, a systematic perturbative analysis is also possible in the parameter $g=1-2 \alpha$. For small $|g| \ll 1$ and for $\omega_{c}$ being the largest energy scale (scaling limit) the SBM can be mapped onto the interacting resonant level model (IRLM),$\stackrel{1,2}{2}$ which describes a quantum dot connected to a lead featuring fermionic degrees of freedom with a constant density of states of bandwidth $2 \omega_{c}$. Its Hamiltonian reads

$$
\begin{aligned}
H_{\mathrm{tot}}=\epsilon d^{\dagger} d & +\sum_{k} \varepsilon_{k} a_{k}^{\dagger} a_{k}+\sqrt{\frac{\Gamma_{0}}{2 \pi \nu}} \sum_{k}\left(d^{\dagger} a_{k}+d a_{k}^{\dagger}\right) \\
& +\frac{U}{2 \nu}\left(d^{\dagger} d-d d^{\dagger}\right) \sum_{k k^{\prime}}: a_{k}^{\dagger} a_{k^{\prime}}:
\end{aligned}
$$

where $d^{(\dagger)}$ is the fermionic dot ladder operator, $a_{k}^{(\dagger)}$ is an annihilation (creation) operator of a fermion in the lead with energy $\epsilon_{k}$, and the density of states in the lead is $\sum_{k} \delta\left(\varepsilon_{k}-\omega\right)=\nu$ for $\omega \ll \omega_{c}$. Normal ordering with respect to (w.r.t.) the reservoir equilibrium distribution is denoted by : $\cdots:$. The IRLM parameters are related to the ones of the SBM as $U=1-\sqrt{2 \alpha}$ and $\Gamma_{0}=\Delta^{2} / \omega_{c}$. Our observable of interest $P(t)$ corresponds to $2\left\langle d^{\dagger} d\right\rangle(t)-1$, with the expectation value of the dot level occupancy $\left\langle d^{\dagger} d\right\rangle(t)$ and the initial condition $\left\langle d^{\dagger} d\right\rangle(0)=1$. Throughout this work we mainly use the language of the IRLM but switch to the SBM language whenever this is useful to describe the underlying physics.

Concerning the relaxation dynamics of $P(t)$ for $\alpha$ close to $\frac{1}{2}$, is has already been shown by an improved NIBA calculation $\underline{10}$ that the incoherent part $P_{\mathrm{bc}}(t)$ has to be changed to

$$
P_{\mathrm{bc}}(t)=-g[1+3 \Theta(-g)] \frac{e^{-\Gamma_{2}^{*} t}}{\left(T_{K} t\right)^{1+|g|}},
$$

i.e. it is also exponentially decaying with a rate $\Gamma_{2}^{*} \approx$ $\Gamma_{1}^{*} / 2$, the prefactor changes to $-4 g=4(2 \alpha-1)$ for $\alpha>\frac{1}{2}$, and the exponent of the algebraically decaying function was predicted to change to $1-g=2 \alpha$ for $\alpha>\frac{1}{2}$. However, a systematic analysis containing all powers $\sim\left[g \ln \left(T_{K} t\right)\right]^{n}$ with $g=1-2 \alpha$ is still missing, i.e. the corrections in $O(g)$ to the exponent can not be trusted so far. The predictions of improved NIBA were recently confirmed in a letter-style paper using RTRG and FRG for the IRLM $\stackrel{11}{\Perp}$ In addition, it was shown that the incoherent part $P_{\mathrm{bc}}(t)$ has the form Eq. (5) for exponentially large times $\left(T_{K} t\right)^{|g|} \gg 1$ only and an analytical formula was proposed valid for all times $T_{K} t \gtrsim 1$. Furthermore, an improved expression for the rate $\Gamma_{2}^{*}$ was presented, and it was shown that the prefactor of the coherent part $P_{\text {pole }}(t)$ has to be changed to $2 \frac{1-g}{1+g}=\frac{2 \alpha}{1-\alpha}, \frac{12}{\text {. }}$ One purpose of this paper is to present more details of the results from RTRG and FRG for the relaxation dynamics close to the coherent-to-incoherent transition at $\alpha=\frac{1}{2}$.

From this discussion, it is obvious that the relaxation dynamics of the unbiased, Ohmic SBM differs from the NIBA prediction for $\alpha \ll 1$ and for $|1-2 \alpha| \ll 1$ and it remains to be seen if similar deficits prevail for other $\alpha$. The RTRG and FRG methods are perturbative renormalization group methods for nonequilibrium systems, and therefore their applicability range for a controlled analysis of the time evolution is restricted to the weakcoupling regime. However, in contrast to bare perturbation theory or the self-consistent Born approximation, as e.g. applied to the Ohmic spin-boson model at small $\alpha, 9,13$ the RTRG and FRG are unique in the sense that they can select systematically all logarithmically diverging terms in the band width $\omega_{c}$ in all orders of perturbation theory, such that renormalized parameters and exponents of power laws for the time evolution can be determined. Other numerical studies for the SBM using a variety of different methods, either being formally exact or selecting certain subclasses of processes in all orders of perturbation theory, have found exponential dependence and substantiated the coherent-to-incoherent crossover but pre-exponential functions were so far not identified unambiguously $\underline{\underline{14}} \underline{\underline{19}}$

For $\alpha$ close to $\frac{1}{2}$, one of the important characteristics to compare is the transition between coherent and incoherent relaxation. In accordance with the standard terminology in the field of dissipative quantum mechanics 
we speak of incoherent dynamics if $P(t)$ is a monotonically decaying function while we refer to coherent behavior if $P(t)$ is nonmonotonic. To understand the effect of the memory on this transition, we study the system's dynamics in two cases: (1) when it relaxes with timeindependent Hamiltonian out of a nonequilibrium product state, and its dynamics is affected by the memory collected over time, and (2) when in addition at time $t_{q}$ some of the Hamiltonian's parameters are changed abruptly so that the behavior of the system at $t>t_{q}$ is influenced by the interplay of the dynamics in the new regime and the memory collected before $t_{q}$. The first case we name the relaxation protocol, implying that the small system and the bath held in thermodynamic equilibrium were decoupled initially [i.e., $\Gamma_{0}=U=0$ or $\Delta=0, \alpha=1 / 2$ for the SBM Hamiltonian Eq. (1)], and at the time $t=0$ the Hamiltonian in Eq. (4) with non-zero $\Gamma_{0}$ and $U$ comes into effect. The quench protocols in addition to the steps of the relaxation protocol imply the sudden change of the Hamiltonian parameters $\epsilon, \Gamma_{0}$, and/or $U$ at time $t_{q}>0$ [in the language of the SBM the parameters $\epsilon, \Delta$, and/or $\alpha$ are quenched; see Eq. (10)]. Particularly interesting is the quench that invokes a transition between the coherent and incoherent regimes of the dynamics. In this case the difference in the time dependencies is particularly clear. The quench dynamics constitutes the main focus of our work. We investigate quenches of the systembath coupling as well as those of the Zeeman splitting of the up and down spin. In addition we leave the framework of the SBM and identify a part of the SBM physics in a bias voltage driven IRLM with two leads. As the standard relaxation dynamics forms the basis for the understanding of the quench ones a detailed study of the former is presented first. In particular, we note that an understanding of the crossover between coherent and incoherent dynamics requires the knowledge of the time dynamics on intermediate time scales, which is accessible by our methods, numerically as well as analytically. Parts of our results for the quench dynamics were earlier published in a letter-style publication. ${ }^{11}$

At $U=0$, that is $\alpha=1 / 2$, the fermionic quantum dot model (4) becomes noninteracting and can be solved exactly (Toulouse limit). For finite interactions we use the RTRG and FRG method. The RTRG was specifically developed as an analytical tool to study the nonequilibrium physics of small interacting quantum systems coupled to noninteracting reservoirs in the weak coupling limit $\underline{3}^{20}$ In particular, the method has the advantage that it can address each individual term contributing to the time evolution and it can provide analytical insight as to what the generic form of the time evolution looks like. .6 .7 The method has already been applied to the stationary properties of the IRLM and to the time evolution for $\epsilon \gg \Gamma_{0} \stackrel{21}{\stackrel{21}{2}}$ In this paper, we will discuss the solution of the RG equations and the time dynamics for the more difficult resonant case $\epsilon=0$. Moreover, we will generalize the RTRG method to the case of arbitrary timedependent Hamiltonians and will discuss in detail the quench dynamics for an abrupt change of some system parameter. The second method applied in this paper is the FRG, which is a very flexible RG method and can be used for open as well as closed quantum many-body systems of different dimensionality $\underline{\underline{4}}$ The practical implementation for the time evolution in the model at hand has been developed in Refs. 22 and 23 . We discuss in detail how the combined use of the two methods provides controlled analytical as well as numerical access to the standard relaxation and the quench dynamics of the IRLM for small $|U|$ that is close to the Toulouse point. The time evolution of the SBM in other parameter regimes and for other nonequilibrium setups was investigated in Refs. 13, 18, 24 30 using a variety of methods.

The paper is structured as follows. In the next section, we introduce the RTRG method essentially without referring to a specific model and explain in detail how it can be extended to time-dependent Hamiltonians, in particular to study the quench dynamics. In Sect. III we discuss the basic steps to obtain FRG flow equations. Next in Sect. IV we apply both RG approaches to the IRLM and study the dynamics within the relaxation and quench protocols. This section contains the RG flow equations to be solved as well as our analytical and numerical results. We conclude with a brief summary in Sect. V] The Appendixes contain technical details on the generalization of the RTRG method to time-dependent Hamiltonians and quantum quenches together with the flow equations and their solutions for the IRLM.

\section{RTRG METHOD}

\section{A. Basic concepts}

The main goal of the RTRG method is to compute the reduced density matrix of a small interacting quantum system coupled to several reservoirs by integrating out the degrees of freedom of the noninteracting bath. From this, the observables of interest can be extracted. The reservoirs are initially decoupled from the interacting system and are held in thermodynamic equilibrium. Wick's theorem, which is applicable for the ladder operators of the bath, allows us to write a diagrammatic series for an effective Liouvillian which determines the time evolution of the reduced density matrix of the local quantum system.$\frac{3}{2}$ For the SBM (Ref. 7) at small $\alpha$ and the $\operatorname{IRLM}^{21}$ at small $|U|$, that is the SBM for $\alpha$ close to $1 / 2$, the perturbative series contains logarithmic divergencies, which can be regularized either by cutting off the Matsubara frequencies characterizing the poles of the Bose/Fermi-functions of the reservoirs ${ }^{3}$ or by using the Laplace variable $E$ as a flow parameter. The latter is exploited in the recently developed E-RTRG method, $\stackrel{8}{=}$ In both cases one obtains RG flow equations, the solution of which gives the effective Liouvillian.

Here in the main text we qualitatively summarize the ideas of the RTRG method and its extension to time- 
dependent Hamiltonians and quenches; for technical details and the precise diagrammatic rules we refer the interested reader to Appendices $\mathrm{A}$ and $\mathrm{B}$.

The time dependence of the reduced density matrix $\rho(t)$ of the local quantum system is determined by the von Neumann equation. Due to the coupling to the bath, the system acquires a memory which is stored in the form of excitations in the leads. This results in a timedependent effective Liouvillian $L\left(t, t^{\prime}\right)$, defined for times $t>t^{\prime}$, which acts in Liouvillian space of the local system and determines the dynamics of the reduced density matrix via the effective von Neumann equation

$$
i \dot{\rho}(t)=\int_{t_{0}}^{t} L\left(t, t^{\prime}\right) \rho\left(t^{\prime}\right) d t^{\prime}
$$

where the coupling of the system to the bath at time $t=$ $t_{0}$ is implied (later on we will set $t_{0}=0$ for convenience). The effective Liouvillian can be decomposed as

$$
L\left(t, t^{\prime}\right)=L_{S}(t) \delta\left(t-t^{\prime}-0^{+}\right)+\Sigma\left(t, t^{\prime}\right)
$$

where $L_{S}(t)$ is the bare Liouvillian of the isolated local quantum system and $\Sigma\left(t, t^{\prime}\right)$ is the dissipative part of the kernel emerging from the coupling to the reservoirs. Defining for $t>t^{\prime}$ a propagator $\Pi\left(t, t^{\prime}\right)$ relating the reduced density matrix at time $t$ to the one at $t^{\prime}$ where system and bath are assumed to be decoupled, we can write the solution of the kinetic equation formally as

$$
\rho(t)=\Pi\left(t, t_{0}\right) \rho\left(t_{0}\right)
$$

where the relation between $\Pi\left(t, t^{\prime}\right)$ and $L\left(t, t^{\prime}\right)$ is given by

$$
\Pi\left(t, t^{\prime}\right)=\theta\left(t-t^{\prime}\right)-i \iint \theta\left(t-t_{1}\right) L\left(t_{1}, t_{1}^{\prime}\right) \Pi\left(t_{1}^{\prime}, t^{\prime}\right) d t_{1} d t_{1}^{\prime} .
$$

Here, all functions depending on two time arguments are retarded ones, i.e., are defined as zero for negative time differences. The last equation expresses the physical property that $-i L\left(t, t^{\prime}\right)$ contains the sum of all correlated processes between $t^{\prime}$ and $t$. The rules for the classification of all these processes by a diagrammatic expansion in the system-bath coupling are provided in Appendix A.

For a time-independent Hamiltonian, the effective Liouvillian is a function of the time difference only. The resulting von Neumann equation

$$
i \dot{\rho}(t)=\int_{t_{0}}^{t} L\left(t-t^{\prime}\right) \rho\left(t^{\prime}\right) d t^{\prime}
$$

can be formally solved by means of a half-sided Fourier transform (or Laplace transform with the rotated Laplace variable $z \rightarrow-i E)$

$$
\begin{aligned}
\rho(t) & =\Pi\left(t-t_{0}\right) \rho\left(t_{0}\right), \\
\Pi(t) & =\frac{1}{2 \pi} \int_{-\infty+i 0^{+}}^{+\infty+i 0^{+}} e^{-i E t} \Pi(E) d E,
\end{aligned}
$$

where

$$
\Pi(E)=\frac{i}{E-L(E)}
$$

is the propagator containing the effective Liouvillian $L(E)=\int_{0}^{\infty} L(t) e^{i E t} d t$ in Laplace representation. The analytical continuation of the propagator can be performed from the real axis into the complex plane. As a response function with $\Pi(t<0)=0, \Pi(E)$ is an analytical function in the upper half-plane. Thus, the Laplace integral transforms into an integral over the contour $\mathcal{C}$ which goes around the nonanalyticities of the propagator in the lower half-plane. The general structure of those has been investigated in Refs. 6 and 7 . In short, the real and imaginary parts of the pole positions $z_{i}=\Omega_{i}-i \Gamma_{i}$ determine the oscillation frequencies $\Omega_{i}$ and decay rates $\Gamma_{i}$ of an exponential decay. Poles with a finite real part occur in pairs $z_{i}^{ \pm}= \pm \Omega_{i}-i \Gamma_{i}$. One pole is always located at $E=0$ corresponding to the stationary state $L\left(i 0^{+}\right) \rho_{\text {stat }}=0$. Additionally, branch cuts occur from the nonanalytic function $L(E)$, which contains typically logarithms or power laws arising from logarithmic divergencies in the perturbative series. For the time evolution they lead to pre-exponential functions which, in the long-time limit $T_{K} t \gg 1$, are typically proportional to $\left(T_{K} t\right)^{-k} F\left(g \ln \left[T_{K} t\right]\right)$ with some model-dependent integer $k=0,1, \ldots$ and a slowly varying logarithmic function $F\left(g \ln \left[T_{K} t\right]\right)$, where $T_{K}$ is a typical low-energy scale (ana$\log$ of the Kondo temperature) and $g$ is a dimensionless coupling constant.

The non-Markovian contribution to the dynamics is encrypted in the $E$ dependence of the effective Liouvillian $L(E) . L(E)$ is computed by means of the RTRG approach. 3.8 As proposed in Ref. 8, it is convenient to decompose the Liouvillian as

$$
L(E)=L_{\Delta}(E)+E L^{\prime}(E)
$$

where $L_{\Delta}(E)$ and $L^{\prime}(E)$ are slowly varying logarithmic functions. The idea of the E-RTRG method, 7,8 where the Laplace variable $E$ is used as the flow parameter, is to set up equations for the derivatives $\partial_{E} L_{\Delta}(E)$ and $\partial_{E} L^{\prime}(E)$, and to resum the series on the right-hand side (r.h.s.) of the differential equation in terms of effective propagators and effective vertices. Closing the set of differential equations by deriving corresponding differential equations for the effective vertices, one obtains universal RG equations in the infinite band-width limit $\omega_{c} \rightarrow \infty$ which are free of logarithmic divergencies. Provided that the effective vertices stay small (the so-called weak-coupling limit), the RG equations can be solved perturbatively in the renormalized couplings along an arbitrary path in the complex plane, providing analytical access to an individual study of all singularities and branch cuts in the lower half of the complex plane.

Once the parts $L_{\Delta}(E)$ and $L^{\prime}(E)$ of the effective Liouvillian are known, the propagator Eq. (12) can be written 
as

$$
\begin{aligned}
R(E) & =-i \Pi(E) \\
& =\frac{1}{E-\tilde{L}_{\Delta}(E)} Z^{\prime}(E)=\tilde{R}_{\Delta}(E) Z^{\prime}(E),
\end{aligned}
$$

where we have defined

$$
Z^{\prime}=\frac{1}{1-L^{\prime}(E)}, \quad \tilde{L}_{\Delta}(E)=Z^{\prime}(E) L_{\Delta}(E),
$$

and

$$
\tilde{R}_{\Delta}(E)=\frac{1}{E-\tilde{L}_{\Delta}(E)} .
$$

For the special case of the IRLM, the RG equations for $\tilde{L}_{\Delta}(E)$ and $L^{\prime}(E)$ together with the results for the effective vertices have been derived in Ref. 21 using the Matsubara cutoff scheme of Ref. 3. The same equations can be obtained within E-RTRG by using the Laplace variable as flow parameter $\stackrel{31}{\underline{1}}$ The results are summarized in Appendix $\mathrm{C}$ and will be used in this work as a starting point to analyze the dynamics within the relaxation and quench protocols.

\section{B. Extension to quenches}

In this paper, we extend the RTRG method to quenches, namely, abrupt parameter changes in the Hamiltonian. The system is described by the Hamiltonian $H_{\mathrm{tot}}^{i}$ before the time of the quench $t_{q}$ and by $H_{\text {tot }}^{f}$ afterwards, while both $H_{\text {tot }}^{i}$ as well as $H_{\text {tot }}^{f}$ are assumed to be time independent. In this case, the integral in Eq. (6) can be split into two parts that describe the memory of the system before and after $t_{q}$. Equation (10) determines the density matrix $\rho_{i}(t)=\rho(t) \theta\left(t_{q}-t\right)$ before the quench, where the Liouvillian $L_{i}$, calculated using $H_{\text {tot }}^{i}$, is taken. In the equation describing the dynamics of the density matrix $\rho_{f}(t)=\rho(t) \theta\left(t-t_{q}\right)$ after the quench there is an additional term containing the memory of the systems dynamics before the quench:

$$
\begin{aligned}
i \dot{\rho}_{f}(t) & =\int_{t_{q}}^{t} L_{f}\left(t-t^{\prime}\right) \rho_{f}\left(t^{\prime}\right) d t^{\prime} \\
& +\int_{t_{0}}^{t_{q}} \Sigma_{f i}\left(t, t^{\prime}\right) \rho_{i}\left(t^{\prime}\right) d t^{\prime},
\end{aligned}
$$

where the Liouvillian $L_{f}$ is calculated using the Hamiltonian $H_{\mathrm{tot}}^{f}$ after the quench, and the kernel $\Sigma_{f i}\left(t, t^{\prime}\right)$ describes the system's memory of processes that took place before the quench. Therefore, $\Sigma_{f i}\left(t, t^{\prime}\right)$ is only defined for $t^{\prime}<t_{q}<t$.

Most conveniently, the solution of Eq. (17) can be written in terms of the propagator $\Pi_{f i}\left(t, t^{\prime}\right)=\theta(t-$ $\left.t_{q}\right) \Pi\left(t, t^{\prime}\right) \theta\left(t_{q}-t^{\prime}\right)$, which connects the density matrix after the quench with the initial density matrix before the quench:

$$
\rho_{f}(t)=\Pi_{f i}\left(t, t_{0}\right) \rho\left(t_{0}\right) .
$$

(a)

(b)

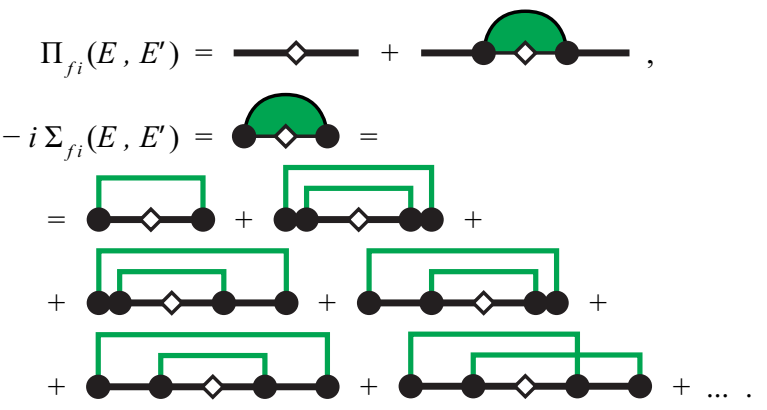

FIG. 1. (Color online) (a) Diagrammatic view of a quench propagator corresponding to Eq. (19) (time representation) or Eq. (22) (Laplace representation). (b) Diagrammatic series for a quench kernel; the first diagrams is expressed in Eq. (24). The thick horizontal lines are propagators of a local system; diamonds are quench vertices; single circles depict tunneling vertices, while doubled circles represent an interaction vertex; the green lines connecting circles correspond to reservoir contractions.

Using Eq. (9), this propagator can be split into two parts

$$
\begin{aligned}
& \Pi_{f i}\left(t, t^{\prime}\right)=\Pi_{f}\left(t, t_{q}\right) \Pi_{i}\left(t_{q}, t^{\prime}\right) \\
& -i \iint \Pi_{f}\left(t, t_{1}\right) \Sigma_{f i}\left(t_{1}, t_{1}^{\prime}\right) \Pi_{i}\left(t_{1}^{\prime}, t^{\prime}\right) d t_{1} d t_{1}^{\prime},
\end{aligned}
$$

where $\Pi_{f / i}\left(t, t^{\prime}\right)=\Pi_{f / i}\left(t-t^{\prime}\right)$ are only defined for $t>t^{\prime}$ and are the propagators of a system time evolved for all times with $H_{\text {tot }}^{i / f}$ taken from Eqs. (11) and (12) with Liouvillian $L_{i / f}$. Inserting Eq. (19) in (18) and using $\Pi_{i}\left(t_{q}-t_{0}\right) \rho\left(t_{0}\right)=\rho\left(t_{q}\right)$ we obtain

$$
\begin{aligned}
& \rho_{f}(t)=\Pi_{f}\left(t-t_{q}\right) \rho\left(t_{q}\right) \\
& -i \iint \Pi_{f}\left(t-t_{1}\right) \Sigma_{f i}\left(t_{1}, t_{1}^{\prime}\right) \Pi_{i}\left(t_{1}^{\prime}-t_{0}\right) \rho\left(t_{0}\right) d t_{1} d t_{1}^{\prime} .
\end{aligned}
$$

The first term describes the dynamics without any memory to times smaller than the quench time, i.e. the density matrix $\rho\left(t_{q}\right)$ at the quench time is used as initial condition for the time evolution after the quench as if the local system and the reservoirs were decoupled up to time $t_{q}$. The second term describes the memory part where the quench time is inside the memory kernel $\Sigma_{f i}$.

Defining the double Laplace transform

$$
\Pi_{f i}\left(E, E^{\prime}\right)=\iint e^{i E\left(t-t_{q}\right)-i E^{\prime}\left(t^{\prime}-t_{q}\right)} \Pi_{f i}\left(t, t^{\prime}\right) d t d t^{\prime},
$$

and analogous for $\Sigma_{f i}\left(E, E^{\prime}\right)$, Eq. (19) can be written in Laplace space as

$$
\Pi_{f i}\left(E, E^{\prime}\right)=\Pi_{f}(E)\left[1-i \Sigma_{f i}\left(E, E^{\prime}\right)\right] \Pi_{i}\left(E^{\prime}\right) .
$$

Using the inverse Laplace transform of the second term on the r.h.s. of this equation and substituting it for the second part of the propagator Eq. (19), we can write the 
time evolution Eq. (20) as

$$
\begin{aligned}
\rho_{f}(t)= & \frac{1}{2 \pi} \int e^{-i E\left(t-t_{q}\right)} \Pi_{f}(E) \rho\left(t_{q}\right) d E \\
& -i \iint e^{-i E\left(t-t_{q}\right)-i E^{\prime}\left(t_{q}-t_{0}\right)} \\
& \times \Pi_{f}(E) \Sigma_{f i}\left(E, E^{\prime}\right) \Pi_{i}\left(E^{\prime}\right) \rho\left(t_{0}\right) \frac{d E d E^{\prime}}{(2 \pi)^{2}} .
\end{aligned}
$$

The two terms of Eq. (22) and the diagrammatic expansion of the memory kernel in terms of the coupling vertices are illustrated in Laplace space in Fig. 1(a) and Fig. 1(b). Here, the quench vertex is indicated as a diamond and is implemented in the diagrammatics as a unit matrix vertex:

$$
\text { quench vertex: } \hat{1}=\diamond \text {. }
$$

As implied by Eq. (22) all operators on the left of the quench vertex depend on the Laplace variable $E$ and have to be taken w.r.t. $H_{\text {tot }}^{f}$, while the ones on the right depend on $E^{\prime}$ and are taken w.r.t. $H_{\text {tot }}^{i}$. The diagrammatic rules are explained in detail in Appendix B] some examples of diagrams are shown in Fig. 1(b) for the IRLM. The diagrams consist of vertices with one or two reservoir lines, corresponding to tunneling and Coulomb interaction vertices, respectively. The vertices are connected by propagators $R=-i \Pi$ (horizontal black lines) and reservoir contractions (green lines). In each diagram the quench vertex has to be inserted in every bare propagator. Analogous to Ref. 8, we resum all diagrams with excitations living only before and after the quench (i.e. contractions which do not cross over the quench) such that full effective propagators and vertices occur to the left and right of the quench vertex. As a consequence only diagrams with contractions crossing over the quench vertex have to be considered. Sorting diagrams by the number of contractions, i.e. the number of excitations gone through the quench, the series starts as shown in Fig. 1(b). In this we present all diagrams with one or two contractions. Using (B12) and A14 and considering the IRLM with only one reservoir, chemical potential $\mu=0$, and zero temperature, the first diagram is explicitly given by (for the notation see Appendices $\mathrm{A}$ and B)

$$
\begin{aligned}
& -i \Sigma_{f i}\left(E, E^{\prime}\right)=\int p^{\prime} f\left(p^{\prime} \bar{\omega}\right) G_{1}^{f}(E) \\
& \quad \times R_{f}(E+\bar{\omega}) R_{i}\left(E^{\prime}+\bar{\omega}\right)\left(G_{i}\right)_{\overline{1}}^{p^{\prime}}\left(E^{\prime}+\bar{\omega}\right) d \bar{\omega}
\end{aligned}
$$

where $f(\omega)=\theta(-\omega)$ is the Fermi function at $T=0, \bar{\omega}=$ $\eta \omega, G_{1}^{f / i} \equiv \sum_{p}\left(G_{f / i}\right)_{1}^{p}$ and $R_{f / i}(E)=1 /\left[E-L_{f / i}(E)\right]$. No frequency cutoff is needed since the integral converges. Neglecting the frequency dependence of the second vertex (giving rise to higher order terms) and closing the integration contour in the upper half of the complex plane we see that only the nonanalytic part of $p^{\prime} f\left(p^{\prime} \bar{\omega}\right)=-\frac{1}{2} \operatorname{sign}(\bar{\omega})+p^{\prime} / 2$ contributes to the integral.
The integral around the branch cut of the sign-function at $\bar{\omega}=i \Lambda, 0<\Lambda<\infty$, leads to

$$
\begin{aligned}
& \Sigma_{f i}\left(E, E^{\prime}\right)=\int_{0}^{\infty}\left(G_{f}\right)_{1}(E) R_{f}(E+i \Lambda) \\
& \quad \times R_{i}\left(E^{\prime}+i \Lambda\right)\left(G_{i}\right)_{\overline{1}}\left(E^{\prime}\right) d \Lambda
\end{aligned}
$$

This result will be used in Sec.IVB to analyze the quench dynamics via Eq. (23). All other diagrams of Fig.1(b) are unimportant. The second diagram is divergent and has to be treated by the E-RTRG method by considering its derivative w.r.t. $E \stackrel{8}{\underline{\underline{B}}}$ However, it contributes only to the dynamics of the off-diagonal terms of the reduced density matrix, which we do not consider here. The integrals over the frequencies in all other diagrams do not diverge and therefore computing those does not require a RG procedure. They can be directly evaluated but lead to higher order terms.

\section{FRG METHOD}

The FRG is a flexible method which allows to tackle a variety of open as well as closed quantum many-body models within the same framework. ${ }^{4}$ In this approach one aims at the one-particle irreducible vertex functions from which observables can be computed. The formulation of the FRG approach in Matsubara space provides direct access to the equilibrium properties of microscopic manybody models. It was used for studying quantum dots, quantum wires and two-dimensional lattice models $\underline{\underline{4}}$ The FRG was extended to Keldysh space and steady-state nonequilibrium properties $32-35$ as well as nonequilibrium time evolution ${ }^{22,23,36}$ was studied. To keep the presentation simple we focus on the Keldysh formalism (used in this paper) when presenting explicit formulas from now on.

In a first step of the derivation of flow equations one introduces a cutoff $\Lambda$ to the free propagator

$$
G_{0} \rightarrow G_{0}^{\Lambda}, \quad G_{0}^{\Lambda=\infty}=0, \quad G_{0}^{\Lambda=0}=G_{0},
$$

which is a $2 \times 2$-matrix in Keldysh contour space. During the RG flow this cutoff is removed and the problem of interest is restored. One takes the derivative of the generating functional $\Gamma^{\Lambda}(\{\bar{\phi}\},\{\phi\})$ for the irreducible vertex functions with respect to $\Lambda$

$$
\begin{aligned}
\dot{\Gamma}^{\Lambda}(\{\bar{\phi}\},\{\phi\})= & \operatorname{Tr}\left[G_{0}^{\Lambda} \partial_{\Lambda}\left[G_{0}^{\Lambda}\right]^{-1}\right] \\
& \mp i \operatorname{Tr}\left[\partial_{\Lambda}\left[G_{0}^{\Lambda}\right]^{-1} \frac{\delta^{2} \mathcal{W}^{c, \Lambda}}{\delta \bar{\eta}^{\Lambda} \delta \eta^{\Lambda}}\right]
\end{aligned}
$$

and expands with respect to the source fields $\bar{\phi}, \phi$. Aside from the usual dependence on the quantum numbers and times, the latter carry an additional index for the upper or lower Keldysh contour. Here, the upper (lower) sign is for bosons (fermions) and we defined the generating 
functional of the connected Green's functions by

$$
\begin{array}{r}
\mathcal{W}^{c, \Lambda}(\{\bar{\eta}\},\{\eta\})=\ln \left[\frac { 1 } { \mathcal { Z } _ { 0 } } \int \mathcal { D } \overline { \psi } \psi \operatorname { e x p } \left\{S_{0}^{\Lambda}-i S_{\mathrm{int}}\right.\right. \\
-(\bar{\psi}, \eta)-(\psi, \bar{\eta})\}]
\end{array}
$$

with the noninteracting partition function $\mathcal{Z}_{0}$ and the noninteracting $S_{0}^{\Lambda}$ and interacting $S_{\text {int }}$ part of the action defining the quantum many body problem. This leads to an infinite hierarchy of flow equations for the vertex functions, with the first three given by

$$
\begin{aligned}
\partial_{\Lambda} \gamma_{0}^{\Lambda} & =\operatorname{Tr}\left[G_{0}^{\Lambda} \partial_{\Lambda}\left[G_{0}^{\Lambda}\right]^{-1}\right]-\operatorname{Tr}\left[G^{\Lambda} \partial_{\Lambda}\left[G_{0}^{\Lambda}\right]^{-1}\right] \\
\partial_{\Lambda} \gamma_{1}^{\Lambda}\left(1^{\prime} ; 1\right)= & \sum_{22^{\prime}} S_{22^{\prime}}^{\Lambda} \gamma_{2}^{\Lambda}\left(1^{\prime} 2^{\prime} ; 12\right) \\
\partial_{\Lambda} \gamma_{2}^{\Lambda}\left(1^{\prime} 2^{\prime} ; 12\right)= & \sum_{33^{\prime}} S_{33^{\prime}}^{\Lambda} \gamma_{3}^{\Lambda}\left(1^{\prime} 2^{\prime} 3^{\prime} ; 123\right) \\
& -\sum_{33^{\prime} 44^{\prime}} S_{33^{\prime}}^{\Lambda} \gamma_{2}^{\Lambda}\left(3^{\prime} 4 ; 12\right) G_{4^{\prime} 4}^{\Lambda} \gamma_{2}^{\Lambda}\left(1^{\prime} 2^{\prime} ; 4^{\prime} 3\right) \\
& -\left[\sum_{33^{\prime} 44^{\prime}} S_{33^{\prime}}^{\Lambda} \gamma_{2}^{\Lambda}\left(1^{\prime} 3^{\prime} ; 14\right) G_{44^{\prime}}^{\Lambda} \gamma_{2}^{\Lambda}\left(2^{\prime} 4^{\prime} ; 23\right)\right. \\
& \quad-\left(1^{\prime} \leftrightarrow 2^{\prime}\right)-(1 \leftrightarrow 2) \\
& \left.\quad+\left(1^{\prime} \leftrightarrow 2^{\prime}, 1 \leftrightarrow 2\right)\right],
\end{aligned}
$$

where we have used

$$
S_{1^{\prime} 1}^{\Lambda}=S^{\Lambda}\left(1^{\prime}, 1\right)=\sum_{22^{\prime}} G_{1^{\prime} 2}^{\Lambda}\left[\partial_{\Lambda}\left[G_{0}^{\Lambda}\right]^{-1}\right]_{22^{\prime}} G_{2^{\prime} 1}^{\Lambda}
$$

and an appropriate multi-index $1,1^{\prime}, \ldots$ denoting the quantum numbers, time, and the contour label. In practical applications to time evolution, one is restricted to the lowest-order (in the two-particle interaction $U$ ) truncation scheme of the resulting coupled differential equations for the vertex functions (see Fig. 2). This results in differential equations for the Keldysh components of the self-energy which are controlled to leading order in the interaction but due to the $\mathrm{RG}$ resummation go beyond plain perturbation theory; e.g. in a variety of applications one obtains power laws with interaction dependent

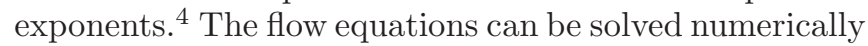
with minor computational effort which allows to study the entire parameter space of a given model with high efficiency. In limiting cases one often even succeeds in gaining analytical insights from these flow equations. Within the time-dependent FRG approach one can straightforwardly treat quenches as all parameters of the Hamiltonian can carry an explicit time dependence. This was discussed in detail in Ref. 36 .

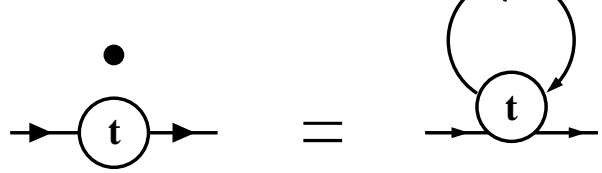

FIG. 2. Diagrammatic representation of the self-energy flow equation. The dot indicates the derivative w.r.t. $\Lambda$ and the slanted line is the single-scale propagator $S^{\Lambda}$.

\section{APPLICATION TO DISSIPATIVE DYNAMICS}

In this section we apply the RG methods introduced above to the IRLM and describe its dynamics. We restrict ourselves to the small- $|U|$ regime corresponding to the SBM close to its coherent-to-incoherent transition. Ultimately, we are interested in the quench dynamics but first present a comprehensive study of the relaxation protocol. It presents the basis for the understanding of the quench protocol.

\section{A. Relaxation protocol}

\section{The RTRG approach}

The general Eqs. (11) and (12) can be used to describe the behavior of the system between the initial time $t_{0}=0$ of coupling the reservoir to the dot and the time of the quench $t_{q}$. Using the form Eq. (14) for the propagator, we obtain

$$
\rho(t)=\frac{i}{2 \pi} \int_{\mathcal{C}} e^{-i E t} \tilde{R}_{\Delta}(E) Z^{\prime}(E) \rho(0) d E,
$$

where $\mathcal{C}$ is a contour enclosing clockwise the lower half of the complex plane including the real axis.

For the particle-hole symmetric case $\epsilon=0$, the $4 \times 4$ matrices $\tilde{R}_{\Delta}(E)$ and $Z^{\prime}(E)$ are provided in Eqs. (C1) and (C6). Since we are only interested in the relaxation of the diagonal matrix elements $\rho_{00}(t)$ and $\rho_{11}(t)$ of the density matrix, we obtain

$$
\begin{aligned}
\left(\begin{array}{c}
\rho_{00}(t) \\
\rho_{11}(t)
\end{array}\right) & =\frac{i}{2 \pi} \int_{\mathcal{C}} e^{-i E t} \\
& \times\left(\frac{\tau_{+}}{E}+\frac{\tau_{-}}{E+i \Gamma_{1}(E)}\right)\left(\begin{array}{c}
\rho_{00}(0) \\
\rho_{11}(0)
\end{array}\right) d E,
\end{aligned}
$$

with $\tau_{ \pm}=\frac{1}{2}\left(1 \pm \sigma_{x}\right)$. Using $\frac{i}{2 \pi} \int_{\mathcal{C}} e^{-i E t} \frac{1}{E}=1$ and $\rho_{00}(0)+\rho_{11}(0)=1$, we find

$$
\begin{aligned}
& \left(\begin{array}{c}
\rho_{00}(t) \\
\rho_{11}(t)
\end{array}\right)=\frac{1}{2}\left(\begin{array}{l}
1 \\
1
\end{array}\right) \\
& \quad+\frac{i}{2 \pi} \int_{\mathcal{C}} \frac{e^{-i E t}}{E+i \Gamma_{1}(E)} \frac{1}{2}\left(\begin{array}{c}
-1 \\
1
\end{array}\right)\left\langle\sigma_{z}\right\rangle(0) d E
\end{aligned}
$$


with $\left\langle\sigma_{z}\right\rangle(0)=\rho_{11}(0)-\rho_{00}(0)$. This gives

$$
\left\langle\sigma_{z}\right\rangle(t)=P(t)\left\langle\sigma_{z}\right\rangle(0),
$$

where

$$
P(t)=\frac{i}{2 \pi} \int_{\mathcal{C}} \frac{e^{-i E t}}{E+i \Gamma_{1}(E)} d E
$$

is defined as the solution for $\left\langle\sigma_{z}\right\rangle(t)$ with the initial condition $\left\langle\sigma_{z}\right\rangle(0)=1$.

To determine $P(t)$ we need the function $\Gamma_{1}(E)$, which follows from the RG equations (C4) and (C5):

$$
\partial_{E} \Gamma_{1 / 2}(E)=-g R_{2 / 1}(E) \Gamma_{1}(E),
$$

where $g=2 U-U^{2}$ and

$$
R_{1}(E)=\frac{1}{E+i \Gamma_{1}(E)}, \quad R_{2}(E)=\frac{1}{E+i \Gamma_{2}(E) / 2},
$$

with the initial conditions $\Gamma_{1 / 2}\left(E=i \omega_{c}\right)=\Gamma_{0}$. For high energies $|E| \gg\left|\Gamma_{1 / 2}(E)\right|$, the solution of the RG equations is

$$
\Gamma_{1 / 2}(E)=\Gamma_{0}\left(\frac{\omega_{c}}{-i E}\right)^{g}=T_{K}\left(\frac{T_{K}}{-i E}\right)^{g},
$$

where

$$
T_{K}=\Gamma_{0}\left(\frac{\omega_{c}}{T_{K}}\right)^{g}=\Gamma_{0}\left(\frac{\omega_{c}}{\Gamma_{0}}\right)^{g /(1+g)}
$$

is the typical low-energy scale which is kept fixed in the scaling limit $\omega_{c} \rightarrow \infty$ and $g, \Gamma_{0} \rightarrow 0$. In this limit, the RG equations (35) cover systematically all orders of $g \ln \left(\omega_{c} / E\right)$ and the exponent of the power law in Eq. (37) is controlled up to $\mathcal{O}\left(U^{2}\right)$. The terms neglected on the r.h.s. of the RG equation for $\Gamma_{1}(E)$ contain terms $\sim U^{3}\left(\Gamma_{1} / E\right)$ of higher orders in $U$ (which can be neglected for small $U$ ) and terms $\sim U\left(\Gamma_{1 / 2} / E\right)^{2}$ of higher order in $\Gamma_{1 / 2} / E$. Integrating the latter from $E=i \omega_{c}$ down to $E \sim i \Gamma_{1 / 2}$ gives a correction to $\Gamma_{1}$ of order $\mathcal{O}\left(U \Gamma_{1 / 2}\right) \sim \mathcal{O}\left(U T_{K}\left[T_{K} / \Gamma_{1 / 2}\right]^{g}\right)$. This is a small correction to Eq. (37) of relative order $U$, which changes only the prefactor of the Kondo temperature $T_{K}$ but does not influence the power law exponent. The FRG approach described above can cover such corrections up to $\mathcal{O}(U)$ since all orders in the tunneling are fully taken into account in each order of the Coulomb interaction.

We note, however, that neglecting higher orders in $\Gamma_{1 / 2} / E$ might imply a change of the power-law exponent of the time evolution in the regime of exponentially large times $T_{K} t \gg 1$ and $\left|g \ln \left(T_{K} t\right)\right| \gtrsim 1$. In this regime one needs a solution of the $\mathrm{RG}$ equations for $E$ exponentially close to a singularity $z^{*}$ of the resolvents $R_{n}(E)$ and it may happen that neglected terms $\sim g\left(\Gamma_{1 / 2}\right)^{2} R_{1}(E) R_{2}(E)$ are of order $g\left|\Gamma_{1 / 2} /\left(E-z^{*}\right)\right|$ since one resolvent is of order $1 /\left|z^{*}\right| \sim 1 /\left|\Gamma_{1 / 2}\right|$ while the other one has a pole at $E=z^{*}$ and is of order

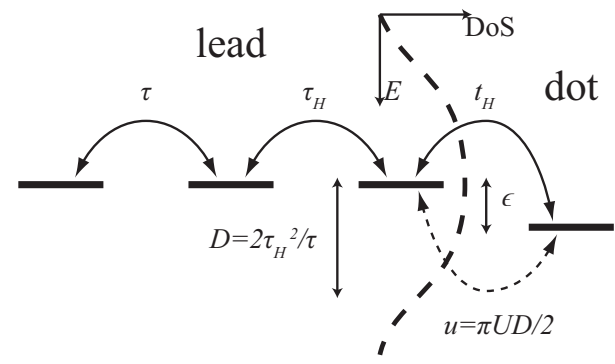

FIG. 3. Quantum dot model considered within FRG.

$1 /\left|E-z^{*}\right|$. Such terms lead to additional logarithmic contributions close to the singularities of the resolvent $R_{1}(E)$ which might result in logarithmic corrections for pre-exponential functions relevant for exponentially large times. Recently, it has been shown ${ }^{7}$ that such behavior can occur for the SBM at small coupling $\alpha$ and it needs to be analyzed whether similar effects may be realized in the IRLM $^{31}$ at small $g$. The most interesting physics occurs when different terms in the dynamics compete. For the relaxation as well as the quench protocols this happens at intermediate $\left(T_{K} t \sim 1\right)$ to long $\left(T_{K} t \gtrsim 1\right)$ times while at exponentially large ones $\left|g \ln \left(T_{K} t\right)\right| \gtrsim 1$ one term dominates (see below). For the former we can safely ignore higher-order terms in $\Gamma_{1 / 2} / E$. We note that for completeness and to make contact to existing results we also analyze our approximate RG equations for exponentially large times keeping in mind that the corresponding result might not present the final answer.

\section{The FRG approach}

In the FRG approach to the IRLM [Eq. (4)], we consider a semi-infinite tight-binding chain, where the first site is tunnel coupled to a single level almost resonant to the Fermi level in the chain (see Fig. 31). A fermion occupying the first site of the chain interacts with the resonant level by a density-density-type interaction of strength $u$. The first lead site and the single level define our quantum dot region. Initially the two sites of the quantum dot region are empty. With the rest of the chain we proceed as follows. We achieve a structureless reservoir by choosing the hopping $\tau$ between the corresponding sites and the hopping $\tau_{H}$ from the second to the first site such that $\tau \rightarrow \infty, \tau_{H} \rightarrow \infty$, but $\tau_{H}^{2} / \tau$ remains constant. As long as the hopping $t_{H}$ between the first site of the chain and the resonant level remains small compared to the bandwidth $\omega_{c}=2 \tau_{H}^{2} / \tau$, we capture the physics of the IRLM as defined in Eq. (4), where the parameters are mapped by choosing $\Gamma_{0}=4 t_{H}^{2} / \omega_{c}$ and $U=2 u /\left(\pi \omega_{c}\right) \underline{\underline{22}, 35}$

The FRG flow equations to be solved numerically were already derived in detail in Ref. 22. The main steps and essential equations are summarized at this point to keep our presentation self-contained. The nonequilibrium problem at hand is tackled by using the Keldysh 
formalism 37,38 First, we determine the retarded (R), advanced (A), and Keldyh (K) components of the Green's function of the decoupled and noninteracting dot region

$$
\begin{aligned}
& g_{i j}^{\mathrm{R}}\left(t, t^{\prime}\right)=-i \Theta\left(t-t^{\prime}\right)\left[e^{-i \hat{\epsilon}\left(t-t^{\prime}\right)}\right]_{i j}, \\
& g_{i j}^{\mathrm{K}}\left(t, t^{\prime}\right)=-i\left[g^{\mathrm{R}}(t, 0) g^{\mathrm{A}}\left(0, t^{\prime}\right)\right]_{i j} .
\end{aligned}
$$

Here and in the following the two sites of the quantum dot region are labeled by the single-particle indices $i, j \in$ $\{1,2\}$. The matrix $\hat{\epsilon}$ appearing in the argument of the exponential function is given by

$$
\hat{\epsilon}=\left(\begin{array}{cc}
t_{H} & \epsilon \\
0 & t_{H}
\end{array}\right) .
$$

We next treat the influence of the reservoir and the interaction separately in the form of self-energies. To obtain the reservoir part, we project the reservoir contribution on the noninteracting dot Green's function $g_{0}$, exploiting that the interaction is restricted to the quantum dot region. This way, the influence of the reservoir is considered by tracing over the particles temporarily (virtually) residing in the reservoir by tunneling from the dot and back. This yields a Dyson equation for the reservoirdressed but noninteracting dot Green's function $G_{0}$ :

$$
G_{0}=g_{0}+g_{0} \Sigma_{\text {res }} G_{0},
$$

where we have left implicit internal matrix multiplications w.r.t. the Keldysh and single-particle indices and convolutions w.r.t. time. If we restrict ourselves to a structureless reservoir, the reservoir contribution to the self-energy $\Sigma_{\text {res }}$ is given by

$$
\begin{aligned}
& \sum_{\mathrm{res}, i j}^{\mathrm{R}}\left(t^{\prime}, t\right)=\left[\Sigma_{\mathrm{res}, i j}^{\mathrm{A}}\left(t^{\prime}, t\right)\right]^{\dagger}=-2 \omega_{c} i \delta\left(t^{\prime}-t\right) \delta_{i, j} \delta_{i, 1}, \\
& \sum_{\mathrm{res}, i j}^{\mathrm{K}}\left(t^{\prime}, t\right)=-\frac{4 \omega_{c}}{\pi} \mathcal{P}\left(\frac{1}{t^{\prime}-t}\right) \delta_{i, j} \delta_{i, 1}
\end{aligned}
$$

Using the Dyson equation (42) is an exact reformulation of the noninteracting problem; all orders in the tunneling to the reservoirs are kept. Next, we consider the Keldysh self-energy $\Sigma$ arising due to the two-particle interaction and again employ the Dyson equation

$$
G=G_{0}+G_{0} \Sigma G
$$

to obtain the full Green's function $G$. The cutoff procedure used in the FRG consists of two independent auxiliary reservoirs coupled to each of our two dot sites via hybridization $\Lambda \stackrel{34}{ }$ Differentiation of the generating functional of the one-particle irreducible vertex functions with respect to $\Lambda$ yields the above-mentioned infinite hierarchy of flow equations for the vertex functions which is still exact. The truncation of this hierarchy is the only approximation within our approach required to derive a closed set of differential equations which can be integrated numerically. We use the lowest truncation order, and the resulting flow equations for the interaction part of the self-energy are given by

$$
\partial_{\Lambda} \Sigma^{\Lambda}\left(1,1^{\prime}\right)=-i \sum_{2,2^{\prime}} S_{2^{\prime} 2}^{\Lambda} \bar{u}_{121^{\prime} 2^{\prime}}
$$

with a multi-index $1=(t, i, p)$ with $p \in\{-1,1\}$ labeling the Keldysh index and

$$
\begin{aligned}
S_{11^{\prime}}^{\Lambda} & =-\sum_{2,2^{\prime}} G_{12^{\prime}}^{\Lambda}\left[\partial_{\Lambda}\left[G_{0}^{\Lambda}\right]^{-1}\right]_{2^{\prime} 2} G_{21^{\prime}}^{\Lambda} \\
& =\partial_{\Lambda}^{*} G_{11^{\prime}}^{\Lambda} .
\end{aligned}
$$

We introduced the star differential operator $\partial_{\Lambda}^{*}$ which acts only on the free Green's function $G_{0}^{\Lambda}$, not on $\Sigma^{\Lambda}$, in the series expansion $G^{\Lambda}=G_{0}^{\Lambda}+G_{0}^{\Lambda} \Sigma^{\Lambda} G_{0}^{\Lambda}+\ldots$. Additionally we define

$$
\begin{aligned}
\bar{u}_{121^{\prime} 2^{\prime}}= & \delta\left(t_{1}-t_{1}^{\prime}\right) \delta\left(t_{1}-t_{2}\right) \delta\left(t_{1}-t_{2}^{\prime}\right) \\
& \times \delta_{p_{1}, p_{1}^{\prime}} \delta_{p_{2}, p_{2}^{\prime}} \delta_{p_{1}, p_{2}}\left(p_{1}\right) \bar{u}_{i_{1} i_{2} i_{1}^{\prime} i_{2}^{\prime}}(t)
\end{aligned}
$$

with the anti-symmetrized two-particle interaction $\bar{u}_{i_{1} i_{2} i_{1}^{\prime} i_{2}^{\prime}}$. As a consequence of the truncation the FRG results are controlled to leading order in the interaction strength $U$. In the RTRG the interaction was expressed in terms of the prefactor $g=2 U-U^{2}$ of the flow equations (35), which in the solution of the latter appears as an exponent. In the FRG this exponent is only captured to leading order. To prevent the inflation of parameters in the following we still use $g$ when discussing FRG results, keeping in mind this restriction to the lowest order. The present FRG procedure was successfully applied to nonequilibrium transport through correlated quantum

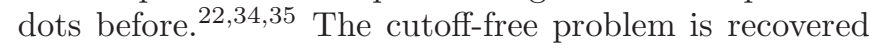
after integrating from $\Lambda=\infty$, where one can give analytic expressions for the vertices, down to $\Lambda=0$, where the problem corresponds to an effective noninteracting one with renormalized time dependent single-particle parameters.

At the point of particle-hole symmetry $\epsilon=0$ for $t \gg \omega_{c}^{-1}$ and to leading order in $g$ the only relevant flow equation is the one for the hopping amplitude between the resonant level and the first site of the reservoir. It reads

$$
\partial_{\Lambda} t_{H}^{\Lambda}(t)=\frac{\pi g}{8 \omega_{c}} i \partial_{\Lambda}^{*} G_{1, l}^{K}(t),
$$

where $\partial_{\Lambda}^{*}$ acts on the free part of the full equal time Keldysh component of the Green function $G_{1, l}^{K}(t)$ only. Its initial condition is $t_{H}^{\Lambda=\infty}(t)=t_{H}$ for all $t$. Employing the projection technique we arrive at

$$
\begin{array}{r}
\partial_{\Lambda} t_{H}^{\Lambda}(t)=i \frac{\pi g \omega_{c}}{4} \partial_{\Lambda}^{*} \int t_{H}^{\Lambda}\left(t^{\prime}\right)\left[g^{>}\left(t, t^{\prime}\right) G^{<}\left(t^{\prime}, t\right)\right. \\
\left.-g^{<}\left(t, t^{\prime}\right) G^{>}\left(t^{\prime}, t\right)\right] d t^{\prime},
\end{array}
$$

with the structureless reservoir's Green functions $g^{>(<)}$ and the single level's Green functions $G^{>(<)}$. The 
greater and lesser functions are related to the retarded, advanced, and Keldysh components by a Keldysh rotation. 37,38

Already in this simple truncation scheme the logarithmic terms present in lowest-order perturbation theory in $g$ are resumed consistently. One obtains a renormalized hopping amplitude featuring a power law with interaction dependent exponent. This exponent is correct to leading order in the interaction. ${ }^{35}$ It was shown that for the time evolution FRG (Ref. 22) leads to terms exponentially decaying in time with interaction-dependent decay rates as well as power-law corrections $t^{-\kappa}$ with $g$-dependent exponent $\kappa$ which is consistent with the one found within RTRG 21

To obtain analytical results using FRG we replace $t_{H}\left(t^{\prime}\right)$ by $t_{H}(t)$ in Eq. (50), which is justified to order $g$. To lowest order in $1 / \omega_{c}$ we then obtain

$$
\frac{\partial_{\Lambda} t_{H}^{\Lambda}(t)}{t_{H}^{\Lambda}(t)}=\frac{g}{2} \partial_{\Lambda}\left[-i \int_{2 / \omega_{c}}^{t} G_{0}^{A, \Lambda}(x) \frac{d x}{x}+\frac{\pi}{2} G_{0}^{K, \Lambda}(t)\right],
$$

with the noninteracting but reservoir dressed advanced Green function $G_{0}^{A, \Lambda}(x)=i \exp \left(-\Gamma_{0} x / 2\right)$ as well as $i G_{0}^{K}(t)=\exp \left(-\Gamma_{0} t\right)$. The high energy cutoff $\tau_{H}^{2} / \tau=$ $\omega_{c} / 2$ has to be introduced by hand as the higher order terms in $1 / \omega_{c}$ were dropped in Eq. (51). Integrating Eq. (51) we find

$$
t_{H}(t)=\frac{\sqrt{T_{K} \omega_{c}}}{2} \exp \left[-\frac{g}{2}\left(\mathrm{E}_{1}\left(\frac{\Gamma_{0} t}{2}\right)+\frac{i \pi}{2} e^{-\Gamma_{0} t}\right)\right]
$$

with $\mathrm{E}_{1}(z)=\int_{z}^{\infty} e^{-x} d x / x$ being the exponential integral. For the initial condition $\rho_{z}(0)=1$ the relaxation dynamics is then given by

$$
\begin{aligned}
P(t) & =G^{R}(t, 0) G^{A}(0, t)+ \\
& +i \iint G^{R}\left(t, t_{1}\right) \Sigma^{K}\left(t_{1}, t_{2}\right) G^{A}\left(t_{2}, t\right) d t_{1} d t_{2},
\end{aligned}
$$

with $G^{R / A}\left(t, t^{\prime}\right)=\mp i e^{\mp \int_{t^{\prime}}^{t} d \tau \Gamma_{0}(\tau) / 2} \theta\left( \pm\left[t-t^{\prime}\right]\right)$ and the self-energy $\Sigma^{K}\left(t_{1}, t_{2}\right)=\Gamma_{0}\left(t_{1}, t_{2}\right) /\left(\pi\left[t_{2}-t_{1}\right]\right)$. We return to this equation when analyzing the behavior for small and asymptotically large $t$. In the former case the time dependent hybridization $\Gamma_{0}(t)=4\left|t_{H}(t)\right|^{2} / \omega_{c}$ matters, while in the latter one must also take into account $\Gamma_{0}\left(t, t^{\prime}\right)=4 t_{H}(t) t_{H}^{*}\left(t^{\prime}\right) / \omega_{c}$.

\section{Dynamics on short times}

In the RTRG method, for short times, such that $T_{K} t \ll 1$, the integral in Eq. (34) is dominated by the large values $|E| \gg\left|\Gamma_{1}\right|$. Therefore one may use the approximation $\Gamma_{1}(E) \approx T_{K}\left(-i E / T_{K}\right)^{-g}$ of Eq. (37) for the relaxation rate, which, since no information on the infrared cutoff is left, is equivalent to the poor man scaling approximation ${ }^{39,40}$ here generalized to the case where the
Laplace variable serves as a cutoff parameter. Expanding the integrand of Eq. (34) in $\Gamma_{1}(E) / E$ and defining the variable $z=E t$ gives the series

$$
\begin{aligned}
P(t) & \approx \sum_{n=0}^{\infty}(-1)^{n}\left(T_{K} t\right)^{n(1+g)} \int_{\mathcal{C}} e^{-i z}(-i z)^{-1-n(1+g)} \frac{d z}{2 \pi} \\
& =\sum_{n=0}^{\infty}(-1)^{n} \frac{\left(T_{K} t\right)^{n(1+g)}}{\Gamma(1+n[1+g])}
\end{aligned}
$$

where $\Gamma(x)$ is the gamma function. With $g \equiv 1-2 \alpha$ this result coincides precisely with the short time dynamics obtained using the noninteracting blip approximation (NIBA) for the SBM $1^{1.2}$ However, one should keep in mind that $\Gamma_{1}(E) \approx T_{K}\left(-i E / T_{K}\right)^{-g}$ is only a good approximation for large $E$, i.e. only the first two terms of the series Eq. (54) can be trusted. Note that if one neglects $g$ in the denominator $\Gamma(1+n(1+g)) \approx$ $\Gamma(1+n)=n$ !, we obtain the series of the exponential function and the result can be written as

$$
P(t) \approx e^{-\left(T_{K} t\right)^{g} T_{K} t}
$$

This coincides with the perturbative result $P(t)=e^{-\Gamma_{t} t}$, where $\Gamma_{t}=\Gamma(i / t)=T_{K}\left(T_{K} t\right)^{g}$ is the poor man scaling solution cut off at $-i E=1 / t$. Solving the poor man scaling RG equation along the imaginary axis, i.e. for $E=i \Lambda$, we see that $\Lambda$ serves as a flow parameter which has to be cut off at $1 / t$. Our finding is consistent with the generic result that the short-time behavior probes the high-energy one in Laplace space and, therefore, can be obtained from poor man scaling equations cut off at the energy scale $1 / t$, see Refs. 6 and 7 .

Up to order $n=1$ the same result can be obtained from the FRG approach. For times $\omega_{c} \gg 1 / t \gg T_{K}$ the second term in Eq. (53) can be neglected and the argument of the exponential integral is small. We can thus replace $\mathrm{E}_{1}(x)=-\gamma-\log (x)$, which leads to

$$
\Gamma_{0}(t)=T_{K} e^{\gamma g}\left(T_{K} t\right)^{g}
$$

for the time dependent renormalized hopping. Here $\gamma$ denotes the Euler constant. Using this the full retarded Green function at small times is given by

$$
G^{R}(t, 0) \approx-i\left(1-\frac{e^{\gamma g}}{2(g+1)}\left(T_{K} t\right)^{g+1}\right),
$$

leading to

$$
P(t)=1-\frac{e^{\gamma g}}{g+1}\left(T_{K} t\right)^{g+1}+\mathcal{O}\left(\left[T_{K} t\right]^{2}\right) .
$$

Here, the power law with interaction dependent exponent is resummed correctly up to $\mathcal{O}(g)$ and the prefactor is determined within the same order.

The power-law scaling of $1-P(t)$ at $T_{K} t \ll 1$ can also be shown for the numerical data obtained by solving the full FRG flow equations (without any additional approximations aside from the lowest-order truncation). 


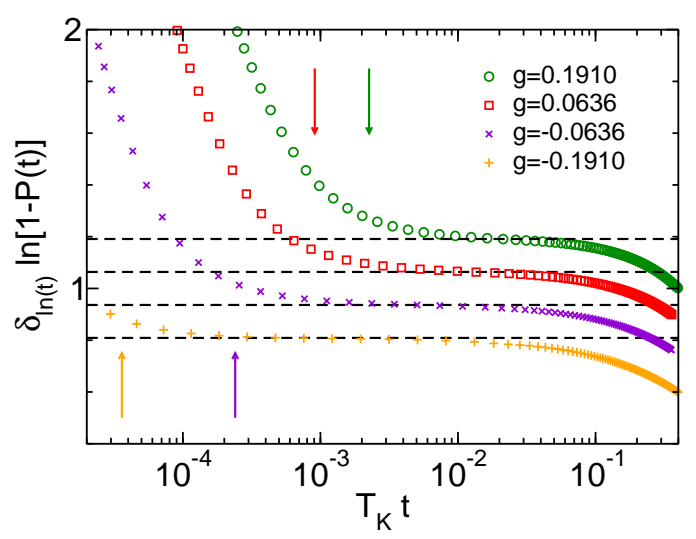

FIG. 4. (Color online) Power law scaling of $1-P(t)$ for $T_{K} t \ll 1$ in the relaxation protocol. The data are produced using the numerical solution of the full FRG flow equations. Symbols represent the time dependence of the logarithmic derivative for different values of $g$. Horizontal dashed lines show the prediction $1+g$ of Eq. (58) for the log-derivative (for the definition of this, see the main text). For $T_{K} t \lesssim 10 T_{K} / \omega_{c}$ band effects start to matter and deviations from power-law scaling appear. The arrows indicate the $g$-dependent $10 T_{K} / \omega_{c}\left(\right.$ with $\left.\omega_{c} / t_{h}=2 \cdot 10^{2}\right)$.

To this end, we numerically compute $\delta_{\ln (t)} \ln [1-P(t)]=$ $d \ln [1-P(t)] / d \ln t$ as centered differences, which becomes a constant if $1-P(t)$ is given by a power law. In Fig. 4. we show $\delta_{\ln (t)} \ln [1-P(t)]$ for different $g$ as a function of $t$ on a log-linear scale. For $T_{K} t \approx 10^{-2}$ the data (symbols) become constant and nicely agree with the exponent predicted by Eq. (58) (dashed lines). The deviations for $t \lesssim 10 / \omega_{c}\left(=5 \cdot 10^{-2} / t_{h}\right.$ in the figure; the arrows indicate $\left.10 T_{K} / \omega_{c}\right)$ are an effect of the reservoirs band width; for such times we leave the scaling limit. Further increasing $\omega_{c}$ in the numerical calculations would move this lower bound to smaller $t$.

The comparison of the results obtained by our two RG approaches and the consistency with the results derived using established methods shows that for small times $T_{K} t \ll 1$ both methods provide controlled access to the relaxation dynamics.

\section{Intermediate to long times}

The relaxation dynamics for times of the order of the typical inverse rates of the system and larger is of particular interest, as it indicates the degree of coherency. For $t \rightarrow \infty$ the dot level becomes half-filled, that is the spin expectation value in the $z$ direction of the SBM vanishes. We say that the system is incoherent if it exhibits a monotonic decay for times $T_{K} t \gtrsim 1$. Nonmonotonicity of $P(t)$, in particular zeros, is characteristic for coherent behavior.

Within RTRG, the dynamics is determined via

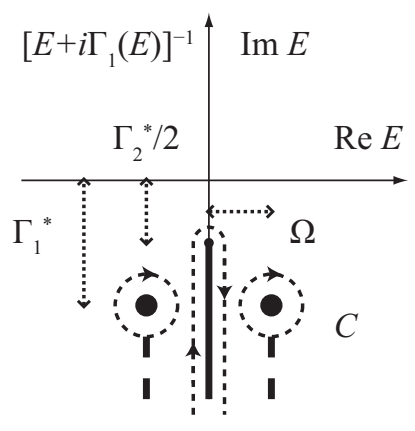

FIG. 5. The nonanalyticities of the propagator $\left[E+i \Gamma_{1}(E)\right]^{-1}$ in Eq. (34) as a function of the complex variable $E$ for positive coupling $g>0$. The main branch cut (thick solid line) and two poles (circles) with attached second order in $g$ branch cuts (thick dashed lines) are shown. The thin dashed line shows the integration contour $\mathcal{C}$ of Eq. (34).

Eq. (34), given by $P(t)=\frac{i}{2 \pi} \int_{\mathcal{C}} d E e^{-i E t} R_{1}(E)$, and follows from the nonanalytical features of the resolvent $R_{1}(E)=1 /\left[E+i \Gamma_{1}(E)\right]$ in the lower half of the complex plane, see Fig. 5 . We find three singularities parametrized by

$$
z_{0}=-i \frac{1}{2} \Gamma_{2}^{*}, \quad z_{ \pm}= \pm \Omega-i \Gamma_{1}^{*},
$$

where the decay rates $\Gamma_{i}>0$ are of the order of $T_{K}$, whereas the oscillation frequency $0<\Omega \sim g T_{K}$. The singularity $z_{0}$ is a branching point followed by a branch cut with a discontinuous $\mathcal{O}(g)$ jump. We choose the position of the branch cut on the negative imaginary axis which turns out to be the most convenient choice to calculate the inverse Laplace transform for the time evolution (see below). $z_{ \pm}$denote the positions of two poles which are followed by two branch cuts with an $\mathcal{O}\left(g^{2}\right)$ jump (which can be neglected in leading order). The pole and branch cut nonanalyticities lead to two terms in the time evolution which we denote by

$$
P(t)=P_{\text {pole }}(t)+P_{\mathrm{bc}}(t) .
$$

The degree of coherence of $P(t)$ is given by the interplay of those terms.

An analytical understanding of the nonanalytical features of $R_{1}(E)$ can be obtained by studying the RG equations (35). We first see from the RG equation for $\Gamma_{2}(E)$ that if $E$ approaches a pole $z_{ \pm}=-i \Gamma_{1}\left(z_{ \pm}\right)$of $R_{1}(E)$, $\Gamma_{2}(E)$ obtains a branch cut with jump of $\mathcal{O}(g)$ starting at $z_{ \pm}$, which, when inserted into the RG equation for $\Gamma_{1}(E)$ leads to a branch cut for $\Gamma_{1}(E)$ with jump of $\mathcal{O}\left(g^{2}\right)$. In addition, when $E$ approaches the pole $z_{0}=-i \Gamma_{2}\left(z_{0}\right) / 2$ of $R_{2}(E)$, we see from the RG equation for $\Gamma_{1}(E)$ that $\Gamma_{1}(E)$ has a branch cut starting at $z_{0}$ with jump of $\mathcal{O}(g)$. To calculate the positions of the singularities up to $\mathcal{O}(g)$, we solve the RG equations by a systematic weak coupling expansion in $g \ll 1$. For $\left|E-z_{i}\right| \sim \mathcal{O}\left(T_{K}\right)$ and $g\left|\ln \left(\left|E-z_{i}\right| / T_{K}\right)\right| \ll 1$ we can expand the solution of Eq. (35) in $g$ and can fix the integration constants by 
comparing with the solution (37) at high energies. Up to $\mathcal{O}(g)$ we obtain

$$
\begin{aligned}
& \Gamma_{1}(E) / T_{K} \approx 1-g \ln \frac{-i E+\Gamma_{2}(E) / 2}{T_{K}}, \\
& \Gamma_{2}(E) / T_{K} \approx 1-g \ln \frac{-i E+\Gamma_{1}(E)}{T_{K}} .
\end{aligned}
$$

Since $g|\ln g| \ll 1$ for $g \ll 1$, we can use these equations for all $g^{2} \lesssim\left|E-z_{i}\right| / T_{K} \sim \mathcal{O}(1)$. This allows for a determination of the positions of $z_{i}$ up to $\mathcal{O}(g)$. We note that $\Gamma_{1 / 2}(E)$ has not been replaced by $T_{K}$ in the argument of the logarithm since it is a priori not clear whether the argument becomes a negative real number of $\mathcal{O}(1)$, i.e., the $\mathcal{O}(g)$ correction of the imaginary part of $\Gamma_{i}(E)$ might be important. However, if $E$ is close to one of the pole positions $i z_{ \pm}=\Gamma_{1}\left(z_{ \pm}\right)$or $i z_{0}=\frac{1}{2} \Gamma_{2}\left(z_{0}\right)$, we can neglect this effect for the corresponding decay rate since we can expand around the pole position and use $\partial_{E} \Gamma_{i} \sim \mathcal{O}(g)$ :

$$
\begin{aligned}
\ln [-i E+ & \left.\Gamma_{2}(E) / 2\right] \\
& \approx \ln \left\{-i\left(E-z_{0}\right)\left[1+i \partial_{E} \Gamma_{2}\left(z_{0}\right) / 2\right]\right\} \\
& \approx \ln \left[-i\left(E-z_{0}\right)\right], \\
\ln [-i E & \left.+\Gamma_{1}(E)\right] \\
& \approx \ln \left\{-i\left(E-z_{ \pm}\right)\left[1+i \partial_{E} \Gamma_{1}\left(z_{ \pm}\right)\right]\right\} \\
& \approx \ln \left[-i\left(E-z_{ \pm}\right)\right] .
\end{aligned}
$$

According to Eqs. (61) and (62) this gives for $E=z_{i}+i \Lambda$, with $0<\Lambda \sim \mathcal{O}\left(g^{2}\right)$, the real value $1-g \ln \left(\Lambda / T_{K}\right)$ for $\Gamma_{1}(E) / T_{K}(i=0)$ or $\Gamma_{2}(E) / T_{K}(i= \pm)$. Therefore, to determine the pole positions $z_{0}$ or $z_{ \pm}$, we set $\Gamma_{1}(E)=T_{K}$ in Eq. (62) or $\Gamma_{2}(E)=T_{K}$ in Eq. (61), respectively, and obtain with $-i z_{0} / T_{K}=-\frac{1}{2}+\mathcal{O}(g)$ and $-i z_{ \pm} / T_{K}=$ $-1+\mathcal{O}(g)$ for the $\mathcal{O}(g)$ correction:

$$
\begin{aligned}
2 i z_{0} / T_{K} & =\Gamma_{2}\left(z_{0}\right) / T_{K} \approx 1-g \ln \left(-i z_{0} / T_{K}+1\right) \\
& \left.\left.\approx 1-g \ln \left(-\frac{1}{2}+1\right) \approx 1+g \ln 2 \approx 2^{g}, \quad(65) / z\right) / T_{K}+\frac{1}{2}\right) \\
i z_{ \pm} / T_{K} & =\Gamma_{1}\left(z_{ \pm}\right) / T_{K} \approx 1-g \ln \left(-i z_{ \pm} / \frac{1}{2}\right) \\
& \approx 1-g \ln \left(-1 \mp i \Omega / T_{K}+\frac{1}{2}\right) \\
& \approx 1+g \ln 2 \pm i \pi g \approx 2^{g} \pm i \pi g .
\end{aligned}
$$

Since $\Omega$ must be positive we see that the equation $E+$ $i \Gamma_{1}(E)=0$ has only a solution for positive coupling $g>$ 0 . We note that $\Omega=0$ is excluded since $E=-i \Gamma_{1}^{*}$ lies on the branch cut of $\Gamma_{1}(E)$. In conclusion, we obtain from Eqs. (65) and (66) the following result for the decay rates and the oscillation frequency up to $\mathcal{O}(g)$ :

$$
\Gamma_{1 / 2}^{*} \approx 2^{g} T_{K}, \quad \Omega \approx \pi g T_{K},
$$

and we find that the poles at $z_{ \pm}$exist only for $g>0$.

If the Laplace variable $E$ lies exponentially close to one of the poles, i.e. $g \ln \left(\left|E-z_{i}\right| / T_{K}\right) \sim \mathcal{O}(1)$, which probes the regime of exponentially large times $g \ln \left(T_{K} t\right) \sim \mathcal{O}(1)$, we can replace $\Gamma_{i}(E) \rightarrow \Gamma_{i}\left(z_{i}\right)$ on the r.h.s. of the RG equations (35). Fixing the integration constants by comparison with the solutions (61) and (62) at intermediate energies, we obtain

$$
\begin{aligned}
& \underline{E \approx z_{0}}: \\
& \Gamma_{1}(E) \approx T_{K}\left[\frac{T_{K}}{-i\left(E-z_{0}\right)}\right]^{g}, \Gamma_{2}(E) \approx 2 i z_{0}, \\
& \underline{E \approx z_{ \pm}}: \\
& \Gamma_{1}(E) \approx i z_{ \pm}, \Gamma_{2}(E) \approx T_{K}\left[1-g \ln \frac{-i\left(E-z_{ \pm}\right)}{T_{K}}\right] .
\end{aligned}
$$

We note that the solutions (37), 61, 68 , 60r $\Gamma_{1}(E)$ at high, intermediate, and exponentially small distances from $z_{i}$ can, in leading order in $g$, be interpolated by the compact expression

$$
\Gamma_{1}(E) \approx T_{K}\left[\frac{T_{K}}{-i\left(E-z_{0}\right)}\right]^{g} .
$$

One can check numerically that, for the special values $E=z_{0}+i \Lambda \pm 0^{+}$, with $\Lambda$ real, this formula holds even for larger values of $g$. Therefore, we will use this result below for the evaluation of the branch cut integral to obtain the incoherent part $P_{\mathrm{bc}}(t)$ of the time evolution. For $E$ exponentially close to the poles $z_{ \pm}$a numerical analysis shows that Eq. (70) is correct for small values of $g$. For larger couplings, it turns out that an improved fit is obtained by using

$$
\underline{E \approx z_{ \pm}}: \quad \Gamma_{1}(E) \approx T_{K}\left[\frac{T_{K}}{-i\left(E-\Gamma_{1}(E) / 2\right)}\right]^{g} .
$$

This equation can be employed for an improved evaluation of the pole position and the residuum of the resolvent $R_{1}(E)$. A straightforward calculation gives the result

$$
\begin{gathered}
\Gamma_{1}^{*} / T_{K}=2^{\frac{g}{1+g}}\left[1+\tan ^{2}\left(\frac{\pi g}{1+g}\right)\right]^{-1 / 2} \\
\Omega=\Gamma_{1}^{*} \tan \left(\frac{\pi g}{1+g}\right)=\Gamma_{1}^{*} \cot \left(\frac{\pi}{2} \frac{1-g}{1+g}\right), \\
\frac{1}{1+\partial_{E} \Gamma_{1}\left(z_{ \pm}\right)}=\frac{1-g}{1+g}
\end{gathered}
$$

The ratio between the oscillation frequency $\Omega$ and the decay rate $\Gamma_{1}^{*}$, the so-called quality factor, was earlier computed using improved NIBA (Ref ${ }^{10}$ ) and a field theoretical approach $\stackrel{41}{*}$ Our result is consistent with the one obtained within those approaches. Employing Eqs. (72) and (74) we obtain directly from Eq. (34)

$$
P_{\text {pole }}(t)=2 \frac{1-g}{1+g} \cos (\Omega t) e^{-\Gamma_{1}^{*} t} \Theta(g) .
$$

For $\Gamma_{2}(E)$ it is more difficult to find interpolation formulas valid for all values of $E$ since this function behaves as a power law for high energies but like a logarithm for $E$ close to $z_{ \pm}$. Inserting Eq. (70) into the RG 
equation (35) for $\Gamma_{2}(E)$, approximating $\Gamma_{1}(E) \rightarrow z_{ \pm}$for $\operatorname{sign}(\operatorname{Re}[E])= \pm$, and taking another derivative, gives the differential equation for a special case of the hypergeometric function

$$
y(1-y) \frac{d^{2} \Gamma_{2}}{d y^{2}}+[1-(1+g) y] \frac{d \Gamma_{2}}{d y}=0,
$$

with $y=\left(z_{ \pm}-E\right) /\left(z_{ \pm}-z_{0}\right)$. In principle this equation can be solved providing an interpolation formula for $\Gamma_{2}(E)$ valid for all $E$ and small $g$. However, since $\Gamma_{2}(E)$ is not needed for an evaluation of the time evolution of the diagonal matrix elements of the density matrix, we do not further discuss this issue. We note, that $\Gamma_{2}(E)$ appearing in the resolvent $R_{2}(E)$ is important for the dynamics of the off-diagonal matrix elements but those cannot be measured: It is impossible to prepare an initial state which is off-diagonal in the charge states.

We next aim at the position of $z_{0}=-i \Gamma_{2}^{*} / 2$ for larger values of $g$. Replacing $\Gamma_{1 / 2}(E) \rightarrow \Gamma_{2}^{*}$ on the r.h.s. of the RG equations (35) provides a very good approximation as can be verified numerically. Based on this we show in Appendix D how an improved solution can be found for $\Gamma_{2}(E)$ close to the imaginary axis for $E=z_{0}+i \Lambda$, with $\Lambda>0$. It leads to the improved formula

$$
\frac{\Gamma_{2}^{*}}{2} \approx T_{K}\left[\frac{\pi g}{2 \sin (\pi g)}\right]^{\frac{1}{1+g}}
$$

We note that although the RG equations (35) are only consistent up to $\mathcal{O}(g)$, we have evaluated the analytical results Eqs. (72), (73), and (77) for the position of the singularities taking higher-order corrections in $g$ into account. This analysis is motivated by the fact that the ratio $\Omega / \Gamma_{1}^{*}$ agrees precisely with previous results from NIBA and field theoretical approaches which are nonperturbative in $g$. Therefore, there is some hope that also the improved result Eq. (77) for $\Gamma_{2}^{*}$ is valid for larger values of $g$. To the best of our knowledge the rate $\Gamma_{2}^{*}$ describing the energy broadening of the local state has so far only been analyzed in leading order in $g$, see Ref. 10 .

Finally, we analyze $P_{\mathrm{bc}}(t)$, given by the branch cut integral [we take $E=z_{0}-i x \pm 0^{+}$with $0<x<\infty$ and use $\left.\Gamma_{1}(E)^{*}=\Gamma_{1}\left(-E^{*}\right)\right]$

$$
\begin{aligned}
& P_{\mathrm{bc}}(t)=\frac{1}{\pi} e^{-\frac{\Gamma_{2}^{*}}{2} t} \\
& \times \operatorname{Im} \int_{0}^{\infty} \frac{e^{-x t}}{\Gamma_{2}^{*} / 2+x-\Gamma_{1}\left(z_{0}-i x-0^{+}\right)} d x,
\end{aligned}
$$

where $\Gamma_{1}\left(z_{0}-i x-0^{+}\right) \approx T_{K}\left(T_{K} / x\right)^{g} e^{-i \pi g}$ is taken from Eq. (70). For intermediate to long times $T_{K} t \gtrsim 1$ it is a very good approximation to replace the slowly varying function $x^{g} \rightarrow t^{-g}$ since the exponential function $e^{-x t}$ in the integrand of Eq. (78) restricts the integration range to the regime $x \sim \mathcal{O}(1 / t)$. This leads to the result

$$
P_{\mathrm{bc}}(t) \approx \frac{1}{\pi} \operatorname{Im}\left\{e^{-\gamma_{t} t} \mathrm{E}_{1}\left(\left[\frac{1}{2} \Gamma_{2}^{*}-\gamma_{t}\right] t\right)\right\},
$$

with $\gamma_{t}=T_{K}\left(T_{K} t\right)^{g} e^{-i \pi g}$ and the exponential integral $E_{1}$.

Combined with $P_{\text {pole }}(t)$ this result covers the limit $g \rightarrow$ 0 , where $\gamma_{t}=T_{K}-i 0^{+} \operatorname{sign}(g)$ and $E_{1}\left(\Gamma_{2}^{*} / 2-\gamma_{t}\right)=$ $-\pi \operatorname{sign}(g)$. This gives together with Eq. (75) $P_{\mathrm{bc}}(t)=$ $-e^{-T_{K} t} \operatorname{sign}(g)$ and $P_{\text {pole }}=2 e^{-T_{K} t} \theta(g)$, leading to the exact result $P(t)=e^{-T_{K} t}$ and $T_{K}=\Gamma_{0}$ for $g=0$.

The contribution from the branch cut integral in the regime $1 \lesssim T_{K} t \ll 1 / g$ for small $|g| \ll 1$ can also be analyzed by observing that the resolvent $R_{1}\left(z_{0}-i x+\right.$ $\left.0^{+}\right)-R_{1}\left(z_{0}-i x-0^{+}\right)$has a sharp Lorentzian peak at $x \sim T_{K} / 2$

$$
\begin{aligned}
& R_{1}\left(z_{0}-i x+0^{+}\right)-R_{1}\left(z_{0}-i x-0^{+}\right) \approx-2 \pi \operatorname{sign}(g) \\
\times & \delta_{T_{K}\left(T_{K} t\right)^{g} \sin (\pi|g|)}\left(x+\frac{1}{2} \Gamma_{2}^{*}-T_{K}\left[T_{K} t\right]^{g} \cos [\pi g]\right),
\end{aligned}
$$

where $\delta_{\eta}(x)=\frac{1}{\pi} \eta /\left(x^{2}+\eta^{2}\right)$. For $t \ll 1 / g$, the peak width is much narrower than the typical scale on which the function $e^{i E t}=e^{-\frac{1}{2} \Gamma_{2}^{*} t} e^{-x t}$ varies, i.e. we can replace the Lorentz peak by a $\delta$-function and get

$$
\begin{aligned}
P_{\mathrm{bc}}(t) & \approx-\operatorname{sign}(g) e^{-T_{K}\left(T_{K} t\right)^{g} \cos (\pi g) t} \\
& \approx-\operatorname{sign}(g) e^{-\Gamma_{1}^{*} t} .
\end{aligned}
$$

Note that this leads to a contribution of $\mathcal{O}(1)$ and not of $\mathcal{O}(g)$.

In contrast, for long times $T_{K} t \gg 1 / g$, we can use the asymptotic expansion $E_{1}(z) \approx e^{-z} / z$ and find from (79)

$$
\begin{aligned}
P_{\mathrm{bc}}(t) & \approx-\frac{\sin (\pi g)}{\pi} \\
& \times \frac{1}{\left|\frac{1}{2} \Gamma_{2}^{*} / T_{K}-\left(T_{K} t\right)^{g} e^{i \pi g}\right|^{2}} \frac{1}{\left(T_{K} t\right)^{1-g}} e^{-\frac{\Gamma_{2}^{*}}{2} t} .
\end{aligned}
$$

Although this term is of $\mathcal{O}(g)$ it will dominate over $P_{\text {pole }}(t)$ Eq. (75) of the time evolution as to leading order in $g$ its decay rate is only half of that of the coherent term.

For exponentially large times $\left(T_{K} t\right)^{|g|} \gg 1$, the branch cut contribution Eq. (82) can be approximated for $|g| \ll$ 1 and $\Gamma_{2}^{*} \approx T_{K}$ by

$$
P_{\mathrm{bc}}(t) \approx-g[1+3 \Theta(-g)] \frac{e^{-T_{K} t / 2}}{\left(T_{K} t\right)^{1+|g|}} .
$$

This result was earlier obtained using improved NIBA. $\underline{\underline{10}}$ Note the power-law correction to the exponential decay already mentioned in the Introduction. We emphasize that this approximation is only justified for times so large that $P_{\text {pole }}(t)$ can safely be neglected compared to the dominating term $P_{\mathrm{bc}}(t)$. Thus, the interesting competition of the branch-cut and pole contributions for $g>0$ on time scales of up to a few $T_{K}^{-1}$ can only be studied by keeping Eq. (79). In fact, it is this competition which for $g>0$ can lead to nonmonotonic, that is coherent, behavior of $P$ on time scales $T_{K} t \sim-\log g-$ on asymptotic ones the dynamics is always monotonic due to the 


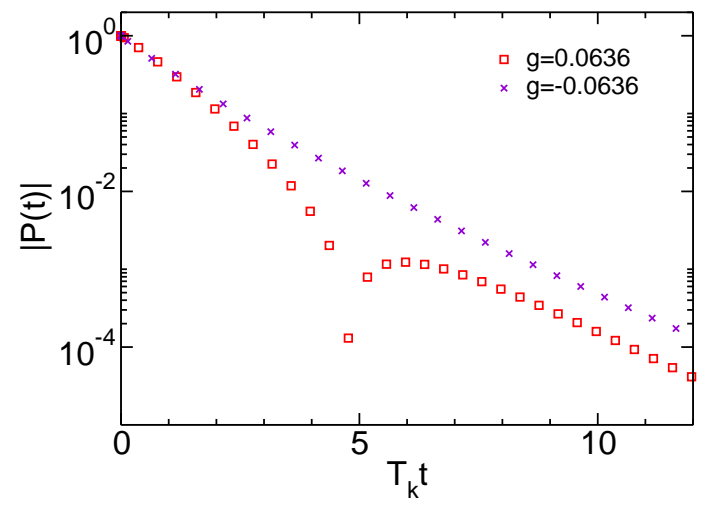

FIG. 6. (Color online) $|P(t)|$ for all time scales. For $g>0$ the competition of the two Eqs. (75) and (779) manifest in the sign change of $P(t)$, appearing as a dip on the logarithmic scale. The partially coherent $(g>0$, red squares) and incoherent $(g<0$, purple crosses $)$ dynamics are compared.

dominating branch cut contribution. On the coherent side of the coherent-to-incoherent transition but close to it the dynamics can thus only be classified as partially coherent. This must be contrasted to the relaxation dynamics of the SBM at small spin-boson coupling $\alpha \ll 1$ and the one of the classical damped harmonic oscillator in its coherent regime (sufficiently weak damping) for which oscillatory behavior can be observed even on asymptotically large times,$\underline{1,2,15}-18$ Analyzing the analytical expressions (75) and (79) for $P(t)$, one finds that up to a certain coupling $g_{1}>0$ only a single local minimum associated with a single transition through zero appears. For $g_{1}<g<g_{2}$, with a certain $g_{2}$, a second zero is found and so on. As our approach is limited to $|g| \ll 1$, we can only speculate about how this behavior crosses over to the coherent behavior with infinitely many zeros obtained at small $\alpha$. From the results (72) and (77), we suspect that beyond a characteristic coupling of order $g \sim 0.4[\alpha=(1-g) / 2 \sim 0.3]$ the finite frequency poles lie closer to the real axis than the branching point, and oscillatory dynamics dominates at large times.

The discussed behavior is confirmed by the full numerical solution of the (approximate) FRG and RTRG flow equations. In Fig. 6] we show $|P(t)|$ obtained from FRG for small $|g|$ on a linear-log scale. For $g>0$ the zero appears as a dip. For negative $g$ there are no poles, so $P_{\text {coh }}=0$. Analyzing Eq. (79) shows that it contains two terms of the same sign both showing exponential decay with the different rates $\Gamma_{1}^{*}$ and $\Gamma_{2}^{*} / 2$. In this regime the dynamics is clearly incoherent; see the crosses in Fig. 6. In Fig. 1 of Ref. 11 we present a detailed comparison of $P(t)$ obtained from the approximate analytical solution of the RTRG flow equations given in Eqs. (75) and (79), as well as the numerical solution of those and the FRG flow equations. This figure also contains a data set from the regime $g_{1}<g<g_{2}$ showing two zeros.
For $g>0$ the asymptotic long time behavior can also be accessed analytically using FRG. For those the second term of Eq. (53) is the dominant one, since it decays slower than the first one (see below). As $\operatorname{Im}[P(t)]=0$ the second term of Eq. (53) only has a nonzero contribution for $\operatorname{Im}\left[\Sigma^{K}\left(t_{1}, t_{2}\right)\right]=\operatorname{Im}\left\{4 t_{H}(t) t_{H}^{*}\left(t^{\prime}\right) /\left[\omega_{c} \pi\left(t_{2}-t_{1}\right)\right]\right\} \neq$ 0 . Therefore we concentrate on this imaginary part, where $t_{H}(t)$ is given by Eq. (52), which leads to

$$
\begin{aligned}
& \operatorname{Im}\left[\Sigma^{K}\left(t_{1}, t_{2}\right)\right]=T_{K} \operatorname{Im}\left\{\exp \left[-\frac{i \pi g}{4}\left(e^{-\Gamma_{0} t_{1}}-e^{-\Gamma_{0} t_{2}}\right)\right]\right\} \\
& \times \frac{1}{\pi\left(t_{2}-t_{1}\right)}+\mathcal{O}\left(g^{2}\right) \\
= & T_{K} \operatorname{Im}\left\{\exp \left[-\frac{i \pi g}{4} e^{-\Gamma_{0} T / 2}\left(e^{\Gamma_{0} \Delta t / 2}-e^{-\Gamma_{0} \Delta t / 2}\right)\right]\right\} \\
& \times \frac{1}{\pi \Delta t}+\mathcal{O}\left(g^{2}\right),
\end{aligned}
$$

with $T=t_{1}+t_{2}$ and $\Delta t=t_{2}-t_{1}$. The integrals in the second term of Eq. (53) are dominated by times $T_{K}(t-$ $\left.t_{1 / 2}\right) \lesssim 1$ such that for $\left(T_{K} t\right)^{|g|} \gg 1$ one can also use $\left(T_{K} t_{1 / 2}\right)^{|g|} \gg 1$ inside the integral. For $t \rightarrow \infty$ we can thus replace $e^{-\Gamma_{0} T / 2}$ in Eq. (84) by its time averaged value over $[t, 2 t]$ and using $\Gamma_{0} \Delta t \propto \Gamma_{0} / T_{K} \ll 1$ find

$$
\operatorname{Im}\left[\Sigma^{K}(\Delta t ; t)\right]=\frac{T_{K}}{\pi \Delta t} \operatorname{Im}\left(\exp \left[-i \frac{\pi g}{2} \frac{\Delta t}{t} e^{-\Gamma_{0} t / 2}\right]\right) .
$$

This self-energy is formally equivalent to one which arises due to a noninteracting reservoir held at chemical potential $\mu_{t}=-\frac{\pi g}{2 t} e^{-\Gamma_{0} t / 2}$, where $t$ can be viewed as a parameter. Using this and $\Gamma_{0} / T_{K} \ll 1$ (which holds for $g>0$ ) Eq. (53) for large times simplifies to Eq. (83). A posteriori we can justify that we only considered the second term of Eq. (53) as it decays exponentially with rate $T_{K} / 2$ while the first term leads to an exponential decay with rate $T_{K}$.

The consistency of all our analytical and numerical results for the relaxation dynamics for intermediate to large times and the agreement to established results in the cases in which a comparison is meaningful confirms that our two RG approaches provide controlled access to the dynamics of the SBM close to the coherent-toincoherent transition.

Finally, we note that for small couplings $g \ll 1$, it is tempting to interpolate the results (81) for $1 \lesssim T_{K} t \ll$ $1 / g$ and (82) for $1 / g \ll T_{K} t$ by taking the sum of both terms. Adding the pole contribution (75) provides the following result for $P(t)$

$$
\begin{aligned}
P(t) & \approx e^{-\Gamma_{1}^{*} t}[2 \cos (\Omega t) \theta(g)-\operatorname{sign}(g)] \\
& -g \frac{1}{\left(\frac{1}{2} \Gamma_{2}^{*} / T_{K}-\left(T_{K} t\right)^{g}\right)^{2}} \frac{1}{\left(T_{K} t\right)^{1-g}} e^{-\frac{1}{2} \Gamma_{2}^{*} t} .
\end{aligned}
$$

This formula can be used for $g \ll 1$ and $T_{K} t \gtrsim 1$ and covers the correct limiting cases of $T_{K} t \ll 1 / g$ and $T_{K} t \gg 1 / g$ since the second term is of $\mathcal{O}(g)$ but decays with a smaller decay rate. Therefore, the first term 
will dominate for $T_{K} t \ll 1 / g$ whereas the second one dominates for $T_{K} t \gg 1 / g$. However, we note that this equation covers the intermediate time regime $T_{K} t \sim 1 / g$ only on a qualitative level. It will be very helpful for a qualitative discussion of the quench protocol considered in the next section.

\section{B. Quench protocols}

The dynamics resulting out of different types of parameter quenches in the IRLM was studied earlier, 15,36,42,43 but no thorough analysis of the role of non-Markovian memory was presented so far.

\section{The RTRG approach}

We begin with adapting the general approach to quenches using the RTRG method described in Sect. II B to the IRLM. Inserting Eq. (24) in Eq. (23) and using the form $R_{f / i}(E)=\tilde{R}_{\Delta}^{f / i}(E) Z_{f / i}^{\prime}(E)$ of the resolvent, we obtain for $t_{0}=0$

$$
\begin{aligned}
\rho_{f}(t) & =\frac{i}{2 \pi} \int e^{-i E\left(t-t_{q}\right)} \tilde{R}_{\Delta}^{f}(E) Z_{f}^{\prime}(E) \rho\left(t_{q}\right) d E-i \int_{0}^{\infty} \iint e^{-i E\left(t-t_{q}\right)} e^{-i E^{\prime} t_{q}} \\
& \times \sum_{\eta= \pm} \tilde{R}_{\Delta}^{f}(E) \tilde{G}_{\eta}^{f}(E) \tilde{R}_{\Delta}^{f}(E+i \Lambda) Z_{f}^{\prime}(E+i \Lambda) \tilde{R}_{\Delta}^{i}\left(E^{\prime}+i \Lambda\right) \tilde{G}_{-\eta}^{i}\left(E^{\prime}\right) \tilde{R}_{\Delta}^{i}\left(E^{\prime}\right) Z_{i}^{\prime}\left(E^{\prime}\right) \rho(0) \frac{d E d E^{\prime}}{(2 \pi)^{2}} d \Lambda,
\end{aligned}
$$

where $\tilde{G}_{\eta}^{f / i}(E)=Z_{f / i}^{\prime} G_{\eta}^{f / i}(E)$. According to Eq. (33), the first term on the r.h.s. gives the following contribution to $\left\langle\sigma_{z}\right\rangle(t)$

$$
P_{f}\left(t-t_{q}\right)\left\langle\sigma_{z}\right\rangle\left(t_{q}\right)=P_{f}\left(t-t_{q}\right) P_{i}\left(t_{q}\right)\left\langle\sigma_{z}\right\rangle(0),
$$

where $P_{i / f}(t)$ is the dot occupancy (spin expectation value) computed without a quench as given in Eq. (34) using the parameters of $H_{\text {tot }}^{i}$ and $H_{\text {tot }}^{f}$, respectively. For the function $P(t)$, this gives the contribution $P_{f}(t-$ $\left.t_{q}\right) P_{i}\left(t_{q}\right)$.

The second term on the r.h.s. of Eq. (87) can be evaluated by inserting the matrix structure of $\tilde{R}_{\Delta}^{f / i}, Z_{f / i}^{\prime}$ and $\tilde{G}_{\eta}^{f / i}$, as provided in Appendix C. Since we are interested in the diagonal elements of the density matrix, a straightforward analysis shows that only the following parts of the various resolvents contribute:

$$
\begin{aligned}
\tilde{R}_{\Delta}^{f / i}(E) & \rightarrow \frac{1}{E+i \Gamma_{1}^{f / i}(E)}\left(\begin{array}{c|c}
\tau_{-} & 0 \\
\hline 0 & 0
\end{array}\right), \\
\tilde{R}_{\Delta}^{f / i}(E+i \Lambda) & \rightarrow \frac{1}{E+i \Lambda+i \Gamma_{2}^{f / i}(E+i \Lambda) / 2}\left(\begin{array}{c|c}
0 & 0 \\
\hline 0 & \mathbb{1}
\end{array}\right) .
\end{aligned}
$$

Neglecting $\Lambda$ in the slowly varying function $Z^{\prime}(E+i \Lambda)$ and inserting the matrix structure of $Z^{\prime}$ and the vertices $\tilde{G}_{\eta}^{f / i}$, we find after straightforward algebra for the part determining the diagonal matrix elements of the density matrix that in Eq. (87) we can employ the replacement

$$
\begin{aligned}
& \sum_{\eta= \pm} \tilde{R}_{\Delta}^{f}(E) \tilde{G}_{\eta}^{f}(E) \tilde{R}_{\Delta}^{f}(E+i \Lambda) Z_{f}^{\prime}(E+i \Lambda) \tilde{R}_{\Delta}^{i}\left(E^{\prime}+i \Lambda\right) \tilde{G}_{-\eta}^{i}\left(E^{\prime}\right) \tilde{R}_{\Delta}^{i}\left(E^{\prime}\right) Z_{i}^{\prime}\left(E^{\prime}\right) \rho(0) \\
& \rightarrow-i 2 U_{i} \sqrt{Z_{f}(E) Z_{i}\left(E^{\prime}\right) \Gamma_{1}^{f}(E) \Gamma_{1}^{i}\left(E^{\prime}\right)} R_{1}^{f}(E) R_{2}^{f}(E+i \Lambda) R_{2}^{i}\left(E^{\prime}+i \Lambda\right) R_{1}^{i}\left(E^{\prime}\right) \frac{1}{2}\left(\begin{array}{c}
-1 \\
1
\end{array}\right)\left\langle\sigma_{z}\right\rangle(0),
\end{aligned}
$$

where $R_{n}^{f / i}(E)=1 /\left[E+i \Gamma_{n}^{f / i}(E) / n\right]$. As a result the contribution of Eq. (87) to the function $P(t)$ can be written as

$$
\begin{aligned}
P(t) & =P^{f}\left(t-t_{q}\right) P^{i}\left(t_{q}\right) \\
& -g_{i} \int_{0}^{\infty} F_{\Lambda}^{f}\left(t-t_{q}\right) F_{\Lambda}^{i}\left(t_{q}\right) d \Lambda,
\end{aligned}
$$

where $g_{i} \approx 2 U_{i}$ in leading order, and the functions $F_{\Lambda}^{i / f}(t)$ are defined by

$$
\begin{aligned}
F_{\Lambda}^{i / f}(t) & =\int e^{-i E t} R_{2}^{i / f}(E+i \Lambda) \\
& \times R_{1}^{i / f}(E) \sqrt{Z_{i / f}(E) \Gamma_{1}^{i / f}(E)} \frac{d E}{2 \pi}
\end{aligned}
$$


and describe the effective emission of a single fermionic excitation by the dot before the quench $\left(F^{i}\right)$ and its absorption after the quench $\left(F^{f}\right)$. The subsequent analytical evaluation of this formula in leading order in $g$ is shown in Appendix E. We note that this analysis is only qualitative, but in essence backed-up by the numerical solution of the full RG equations, since we need results in the intermediate-time regime either for $T_{K}^{f}\left(t-t_{q}\right) \gtrsim 1 / g_{f}$ or for $T_{K}^{i} t_{q} \gtrsim 1 / g_{i}$, where Eq. (86) for the relaxation protocol is only qualitative. However, these interpolation formulas are sufficient for a discussion of the competition between the two terms of $P(t)$ in Eq. 88a describing the effects without and with memory from the dynamics before the quench.

\section{The FRG approach}

In a two-lead geometry, quenches of the single-particle parameters (but not the two-particle interaction $U$ ) of the IRLM were earlier studied in Ref. 36 using FRG. The focus was on systems driven by an applied bias voltage. Within the FRG approach also the interaction can carry an arbitrary time dependence, such that the approach developed in this paper can directly be applied to the problem of present interest.

\section{Interaction quenches}

To illustrate the physics of a quench, which is substantially different from the one of the simple relaxation process, we initially study the instantaneous transition between the (partially) coherent $(g>0)$ and the incoherent $(g<0)$ regimes, keeping the absolute value $|g|$ fixed. The characteristic energy scales $T_{K}^{i / f}$ before and after the quench differ by orders of magnitude. As a consequence, the new small parameter $A^{2}$ appears which is given by the ratio of the smaller Kondo temperature at negative coupling and the larger one at $g>0$. Substituting the expressions for $T_{K}^{i / f} \approx \Gamma_{0}\left(\omega_{c} / \Gamma_{0}\right)^{g_{i / f}}$ from Eq. (38) for $g \ll 1$ we obtain $A \approx\left(\Gamma_{0} / \omega_{c}\right)^{|g|}$.

a. Coherent to incoherent quench. We first consider a quench from $g_{i}=g>0$ to $g_{f}=-g<0$. The dynamics of the system in the (partially) coherent regime implies at least one local minimum and one zero of $P(t)$ in the absence of a quench. The position of the first zero of the relaxation protocol will be denoted as $t^{*}$ in the following. In the absence of the memory term from the quench, obtained within RTRG by leaving out the second term in Eq. 88a,$P(t)$ after the quench is monotonically decaying to zero. In the systems memory from the dynamics before the quench, that is the second term of Eq. (88a), however, the tendency to change the sign of $P(t)$, is kept even at $t>t_{q}$. Thus, if the quench is performed at time $t_{q}<t^{*}$, nonmonotonic behavior appears for $t>t_{q}$ for which the time evolution is already performed with a negative coupling. In Appendix E we show that Eq. (88) can be written as

$$
\begin{aligned}
& P(t) \approx e^{-\Gamma_{1}^{* f}\left(t-t_{q}\right)-\Gamma_{1}^{* i} t_{q}}\left[2 \cos \left(\Omega_{i} t_{q}\right)-1\right]- \\
& -g A S_{T_{K}^{f} t}^{-} S_{T_{K}^{i}}^{+} \frac{e^{-\Gamma_{2}^{* f}\left(t-t_{q}\right) / 2-\Gamma_{2}^{* i} t_{q} / 2}}{T_{K}^{f} t},
\end{aligned}
$$

where $A=\sqrt{T_{K}^{f} / T_{K}^{i}}$ and the function $S^{ \pm}$is defined as

$$
S_{x}^{ \pm}=\frac{(x / 2)^{ \pm g / 2}}{(x / 2)^{ \pm g}-1 / 2} .
$$

Note that we have included for $P^{f}\left(t-t_{q}\right) P^{i}\left(t_{q}\right)$ only the first term of Eq. (86). The contributions of $\mathcal{O}(g)$ resulting from the second term of Eq. (86) lead to a stronger decay compared to the second term of (89). The first term of Eq. (89), corresponding to the coherent contribution, and the second one, describing the system memory, have different signs for $t_{q}<t^{*}$, and therefore compete. If $2 \cos \left(\Omega_{i} t_{q}\right)-1 \gg g$, then, right after the quench the first term dominates, but, due to the twice larger decay rate, becomes recessive later. This leads to a local minimum and a sign change after the quench. With $t_{q}$ approaching $t^{*}, 2 \cos \left(\Omega_{i} t_{q}\right)-1$ decreases and the time scale at which the second term dominates approaches $t_{q}$ from above. For times $t_{q}>t^{*}$, the first one is already negative, so both terms just add up and no local minimum or zero of $P(t)$ appears. In the lower panel of Fig. 7 the absolute value of Eq. [89] is shown on a linear-log scale for fixed $g_{i}=0.1$, different $t_{q}$, and the times of validity of this expression (see above).

The analytical prediction of Eq. (89) is confirmed by the numerical solutions of the FRG and RTRG flow equations. The corresponding $|P(t)|$ are shown in the upper panel of Fig. 7 (symbols: FRG; lines: RTRG). If $t_{q}$ (indicated by the vertical dotted lines) is larger than $t^{*}$ (given by the dip position of the blue curve with $t_{q}$ to the right of the dip), $P(t)$ after the quench does not show a local minimum and/or zero (blue curve). In the opposite case (green and yellow) nonmonotonic behavior is transferred deep into the regime in which the time evolution is performed with a negative $g$. For the case in which $t_{q}$ is significantly to the left of $t^{*}$ (yellow curve) the FRG and the RTRG data show sizable differences at large $t$. In this case the zero of $P(t)$ is transferred to very large times, that is deeply into the regime in which the time evolution is performed with a negative coupling constant. At those times the overall exponential decay already suppressed $|P(t)|$ to a very small value (of the order $10^{-6}$ in the figure). Therefore any small difference in the $P(t)$ obtained by the two approximate methods leads to a drastically different appearance of the curves (e.g. a sizable shift of the zero) on the linear-log scale of the plot.

b. Incoherent-to-coherent quench. We next study the opposite interaction quench with $g_{i}=-g<0$ to $g_{f}=g>0$ and show that monotonic behavior may prevail even after the quench. Again the non-Markovian memory is responsible for this surprising behavior. To 


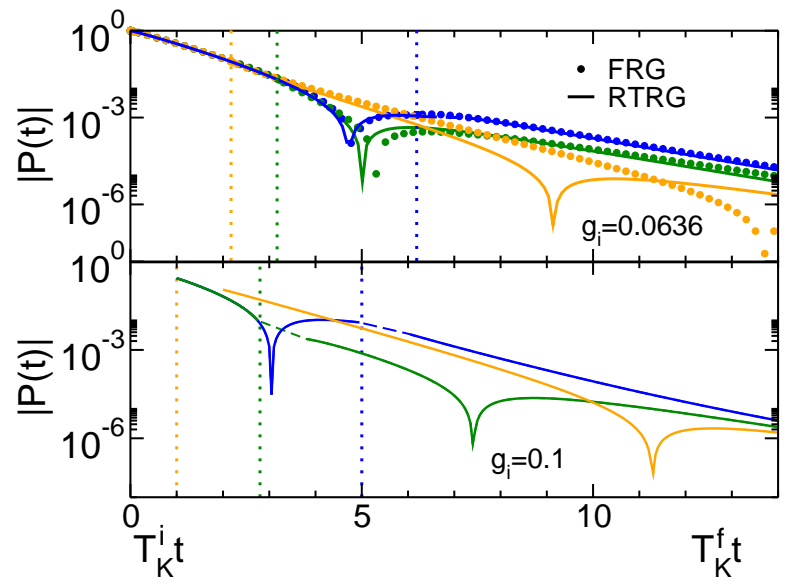

FIG. 7. (Color online) Time evolution of the spin expectation value $P(t)$ in the quench protocol. The coupling is quenched from $g_{i}>0$ to $g_{f}=-g_{i}<0$. The time of the quench $t_{q}$ is varied as indicated by the vertical dotted lines. If $t_{q}$ is smaller than the time $t^{*}$ of the first zero of $P(t)$ in the absence of the quench the non-Markovian memory transfers the nonmonotonic behavior to the time regime after the quench in which the time evolution is performed with a negative coupling constant. Note that the time axis is chosen to be $T_{K}^{i} t$ before the quench and $T_{K}^{f} t$ after it. Since $T_{K}^{i}$ and $T_{K}^{f}$ can differ by orders of magnitude scaling the time axis in this way is physically meaningful. Upper panel: $P(t)$ obtained from the numerical solution of the FRG (symbols) and RTRG (lines) flow equations. Lower panel: the analytical RTRG result Eq. (89). Curves are shown only for times $T_{K}^{f}\left(t-t_{q}\right) \gtrsim 1$ for which this result is applicable.

gain analytical insights we consider Eq. (88) in the limit $T_{K}^{i} t_{q} \gg T_{K}^{f}\left(t-t_{q}\right) \gtrsim 1$. As described in detail in Appendix $\mathrm{E}$, we obtain the approximate expression

$$
\begin{aligned}
& \frac{P(t)}{P\left(t_{q}\right)}=\left[1-\frac{2 A}{S_{T_{K}^{i} t_{q}}^{-}}\right] e^{-\Gamma_{1}^{* f}\left(t-t_{q}\right)} \\
& \times\left[2 \cos \left\{\Omega_{f}\left(t-t_{q}\right)\right\}-1\right]+A \frac{S_{T_{K}^{f} t_{q}}^{+}}{S_{T_{K}^{i} t_{q}}^{-}} e^{-\Gamma_{2}^{* f}\left(t-t_{q}\right) / 2},
\end{aligned}
$$

with $A=\sqrt{T_{K}^{i} / T_{K}^{f}}$. We left out the second term of Eq. (86) for the calculation of $P^{f}\left(t-t_{q}\right)$ in the first term of Eq. 888 since it leads to a subleading contribution $\mathcal{O}\left(g_{f}\right)$ to Eq. (91). We are not interested in exponentially large times and can thus set $S^{ \pm}=2$ in Eq. (91). It simplifies to

$$
\begin{aligned}
\frac{P(t)}{P\left(t_{q}\right)} \approx & (1-A) e^{-\Gamma_{1}^{* f}\left(t-t_{q}\right)}\left[2 \cos \left\{\Omega_{f}\left(t-t_{q}\right)\right\}-1\right] \\
& +A e^{-\Gamma_{2}^{* f}\left(t-t_{q}\right) / 2}
\end{aligned}
$$

This expression shows that because of $A \approx\left(\Gamma_{0} / \omega_{c}\right)^{|g|}$, the oscillatory term of the dynamics after the quench is suppressed with decreasing $g$. A critical $g_{c}$ is found such that for $g<g_{c}$ the relaxation is monotonic after the quench, even though the time evolution is performed with a positive coupling constant. This result is again confirmed by FRG calculations revealing the independence of the qualitative behavior of $P(t)$ from the time of the quench $t_{q}$ (see Fig. 8). We here refrain from showing results obtained from the numerical solution of the RTRG equations and just mention that those are consistent with the behavior described above.

The transfer of monotonic dynamics across the quench can be explained as follows. In the case of the relaxation protocol with $g>0$ there are two competing terms with different relaxation rates and prefactors, which result from the pole $\sim e^{-\Gamma_{1}^{* f}\left(t-t_{q}\right)}\left[2 \cos \left\{\Omega_{f}\left(t-t_{q}\right)\right\}-1\right]$ and branch cut $\sim g e^{-\Gamma_{2}^{* f}\left(t-t_{q}\right) / 2}$ contributions. The latter follows from the non-Markovian terms in the von Neumann equation, and therefore represents the memory of the system. For the sake of simplicity of the qualitative explanation we here use the simplified expressions omitting the power-law dependencies. Due to the small prefactor $g$, the oscillating term always dominates at times $T_{K}^{f}\left(t-t_{q}\right) \sim 1$, but due to the twice larger decay rate is subdominant at larger times. After the quench, the memory term remains the same, i.e., $\sim g e^{-\Gamma_{2}^{* f}\left(t-t_{q}\right) / 2-\Gamma_{2}^{* i} t_{q} / 2}$, where the exponent contains the contributions collected starting at $t=0$. The contribution without memory from the dynamics before the quench, which is equal to $e^{-\Gamma_{1}^{* f}\left(t-t_{q}\right)}\left[2 \cos \left\{\Omega_{f}\left(t-t_{q}\right)\right\}-1\right]$, where $t-t_{q}$ is the time passed after the quench, needs to be multiplied by the value of the density matrix at $t_{q}$, which is $g e^{-\Gamma_{2}^{* i} t_{q} / 2}$. Thus, the competing terms after the quench are $\sim g e^{-\Gamma_{1}^{* f}\left(t-t_{q}\right)-\Gamma_{2}^{* i} t_{q} / 2}\left[2 \cos \left\{\Omega_{f}\left(t-t_{q}\right)\right\}-1\right]$ and $\sim g e^{-\Gamma_{2}^{* f}\left(t-t_{q}\right) / 2-\Gamma_{2}^{* i} t_{q} / 2}$, and, depending on more subtle prefactors [see Eq. (92)], the nonoscillatory term may dominate even right after the quench.

\section{Quench of the level position}

Up to now we exclusively considered the case of vanishing level energy $\epsilon=0$ or, for the SBM, vanishing Zeeman splitting in both the relaxation as well as the quench protocols. We next study the response of the system to abruptly changing $\epsilon$ for $g \geq 0$. For this we only use the flexible FRG approach in which finite $\epsilon$ can straightforwardly be included. We start out with the case of a quench from $\epsilon=0$ to a value much larger than $T_{K}$. The instantaneous increase of the level position from zero, that is resonance, to a large value results in a behavior of $P(t)$ similar to the one observed in the relaxation protocol with a time independent large $\epsilon$; for details see Refs. 21 and 22. For a finite Zeeman field the spin expectation value does no longer vanish at large times (the level occupancy does not approach $1 / 2)$. In Fig. 9 showing $P(t)$ for different $g$ we thus subtracted the stationary value $P_{\text {stat }}$. Qualitatively the dynamics can be described by considering two main contributions. The nonoscillatory term, $e^{-\Gamma_{1}^{*}\left(t-t_{q}\right)}$ stem- 


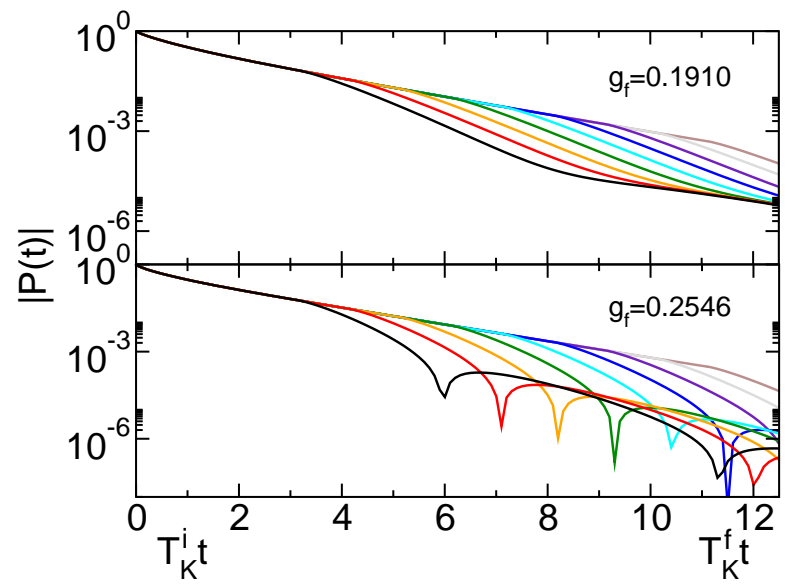

FIG. 8. (Color online) Time evolution of the spin expectation value $P(t)$ in the quench protocol obtained from the numerical solution of the FRG flow equations. Here the coupling is quenched from $g_{i}<0$ to $g_{f}=-g_{i}>0$. Note that the time axis is chosen to be $T_{K}^{i} t$ before the quench and $T_{K}^{f} t$ after it. Different lines correspond to different quench times $t_{q}$ increasing from left to right. Upper panel: $g_{f}<g_{c}$, that is the time evolution even after the quench is monotonic even though it is performed with a positive coupling constant. The non-Markovian memory heavily affects the dynamics after the quench. The qualitative behavior is independent of $t_{q}$. Lower panel: $g_{f}>g_{c}$; nonmonotonic behavior is found after the quench. This is again independent of $t_{q}$.

ming from a pole on the imaginary axis and the term $\left(T_{K} / \epsilon\right) e^{-\Gamma_{1}^{*}\left(t-t_{q}\right) t / 2} \sin \left[\epsilon\left(t-t_{q}\right)\right] /\left[\epsilon\left(t-t_{q}\right)\right]$ originating from a pair of branch cuts positioned away from the imaginary axis. Initially, the second term is suppressed due to the small prefactor $T_{K} / \epsilon t$, so that only weak oscillations on top of an exponential decay are observed, (see Fig. (9). At larger times, $T_{K}\left(t-t_{q}\right) \gtrsim 2 \log \left(\epsilon / T_{K}\right)$, due to the twice smaller decay rate the second term dominates, revealing a weaker decay which is overlaid by (relatively) strong oscillations.

Results for $P(t)$ for the reversed quench from large $\epsilon$ to zero are presented in Fig. 10, For $g>0$ the behavior of the spin expectation value after the quench (lines in Fig. 10) resembles the one found within the relaxation protocol in the (partially) coherent regime (symbols in Fig. (10). However, there is a substantial difference between the two cases for $g=0$. In the quench protocol at large $t$ the decay with the relaxation rate $\Gamma_{0} / 2$ dominates which is absent in the relaxation protocol. This effect can be understood from the analytical expression for $P(t)$ after an $\epsilon$-quench at $g=0$, which is given in Ref. 15.

Non-Markovian memory plays a subdominant role for those types of quenches.

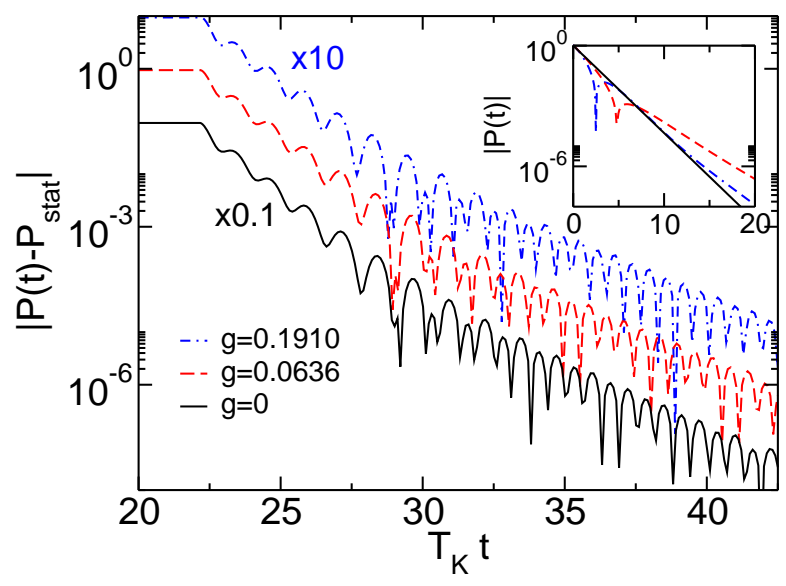

FIG. 9. (Color online) Time evolution of the spin expectation value $P(t)$ after a quench of the Zeeman splitting from zero to $\epsilon / T_{K}=5$ obtained within FRG. The stationary value $P_{\text {stat }} \neq$ 0 was subtracted and the data are presented on a linear-log scale. The behavior for systems with different couplings $[g=$ 0.0636 (dashed red line) and $g=0.1910$, (dotted-dashed blue line)] does not significantly differ from the noninteracting case (black solid line). The inset shows $|P(t)|$ before the quench.

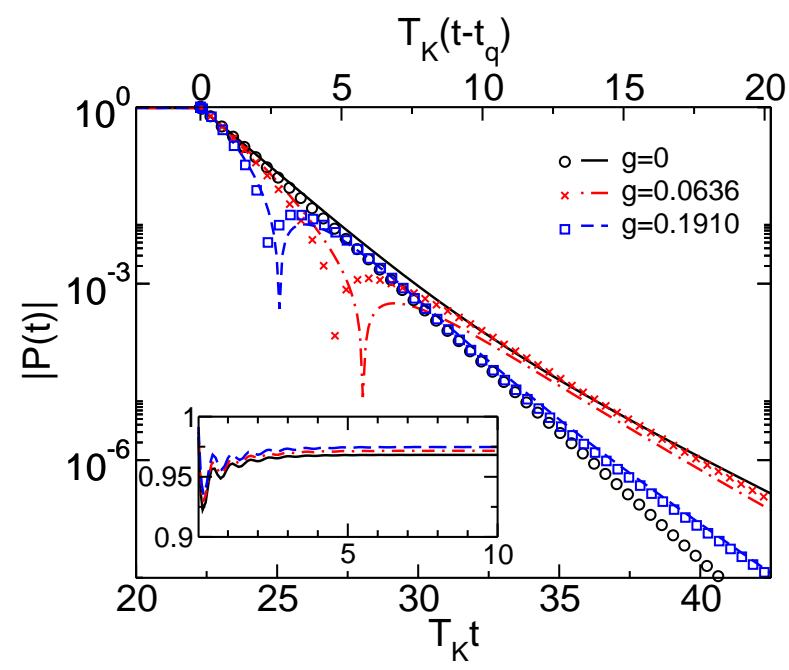

FIG. 10. (Color online) Time evolution of the spin expectation value $P(t)$ after a quench of the Zeeman splitting from $\epsilon / T_{K}=10$ to zero for different $g$ (lines) obtained within FRG. For comparison $|P(t)|$ of the relaxation protocol is shown (symbols). Inset: $P(t)$ before the quench at $T_{K} t_{q}=22.5$.

\section{Current in a two-reservoir setup with level position quench}

The developed methods can be easily generalized for the problem of transport through a two-lead quantum dot, where the current in the reservoirs can be measured. The following setup is considered: at $t=0$ the dot level with large $\epsilon$ is coupled to the reservoirs held at different chemical potentials $\mu_{L / R}= \pm V / 2$ and the time evolution is performed. After relaxation into the stationary state, 
$\epsilon$ is quenched to a value making it resonant with the left lead. Due to the finite voltage $V$ applied across the dot, the model can no longer be mapped onto the SBM. We still discuss the time evolution of this model in this paper as it can partly be understood in terms of the SBM physics described above.

Typical data for the current in the left (upper panel) and right lead (lower panel) in this setup are shown in Fig. 11. The surprising finding is that despite the large bias $V / T_{K}=10$ the left lead current only has a slight trace of oscillations with frequency of the reservoir bias $V$. In two lead setups with finite bias voltage, the latter usually dominates the current (see Refs. 21 and 22); in the present case this can be inferred from the right current (dashed blue line in the lower panel). Instead, the oscillatory part of the left current is characterized by the SBM frequency $\Omega$ appearing for $g>0$; see the dip at $T_{K} t \approx 17.5$ in the dashed blue line of the upper panel of Fig. 11. For small $g, \Omega$ is proportional to $g$ and thus zero for $g=0$ (solid black line in the upper panel). This is a clear evidence that depending on the precise setup, the largest low-energy scale, in the present case $V$, may not serve as a cutoff of the RG flow and is thus not visible in all observables.

The additional dip in the left lead current right after the quench at $T_{K} t_{q}=11.75$ reflects the power-law decay of $P(t)$ in the relaxation protocol at small times given in Eq. (58). During the relaxation process, the tunnel coupling to the left lead, which is in resonance with the dot level, is dominating over the right one, that is, fermions predominantly hop from the dot level to the left lead and back. The left current can thus be approximated as $J_{L}(t) \approx \partial_{t} P(t)$, with $P(t)=2\left\langle d^{\dagger} d\right\rangle(t)-1$, and the time dependence of the current for short times is $\propto t^{g}$. This leads to the first dip right after the quench in the upper panel of Fig. 11.

For large times $T_{K} t \gtrsim 17$ the right current shows oscillations which do not involve transitions through zero; see the dashed blue line in the lower panel of Fig. 11 which becomes smooth for $T_{K} t \gtrsim 17$. Those are absent if one computes the right current with the level being initially resonant with the left lead and no additional parameter quench at a later time (relaxation protocol); see the lower inset. In this case dips appear which are associated to zeros of the current. This difference can be explained as follows. In the quench protocol in addition to the oscillating term with decay rate $\Gamma_{2}^{*} / 2,\left(T_{K} / \epsilon\right) e^{-\Gamma_{2}^{*} t / 2} \sin (\epsilon t) /(\epsilon t)$ a monotonic contribution with the same rate $\propto e^{-\Gamma_{2}^{*} t / 2}$ must appear. Adding those up leads to the observed exponential decay with rate $\Gamma_{2}^{*} / 2$ overlaid by oscillations with frequency $\epsilon$. This monotonic term is absent in the relaxation protocol and can thus be attributed to the system memory from before the $\epsilon$-quench.

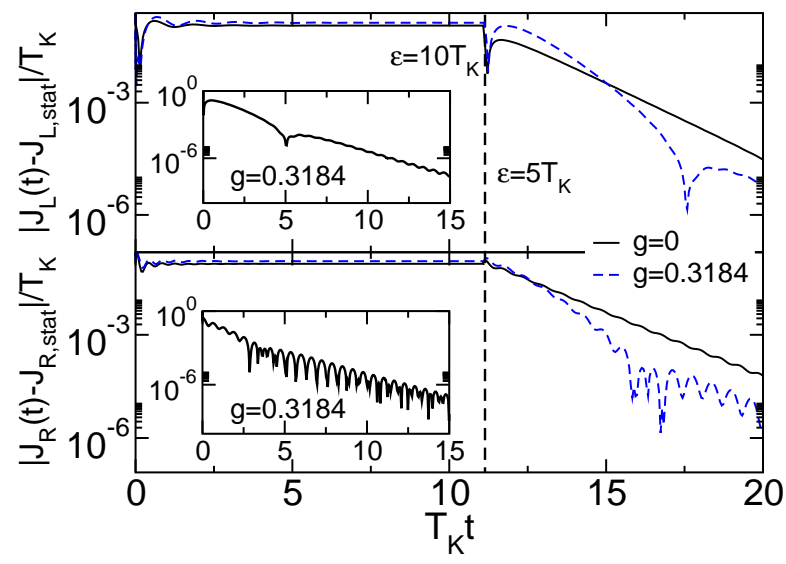

FIG. 11. (Color online) Time dependence of the left (upper panel) and right (lower panel) currents in a two-lead setup with a bias voltage $V / T_{K}=10$ applied symmetrically across the dot. At time $T_{K} t_{q}=11.75$, that is after reaching the stationary state with respect to the initial preparation, the level position $\epsilon$ is abruptly lowered from $10 T_{K}$ to that being resonant with the left lead $\epsilon-V / 2=0$. Black solid lines show the noninteracting case, while blue dashed lines are FRG data obtained for $g=0.3184$. Insets: Time evolution of the left and right currents with $\epsilon-V / 2=0$ initially and no parameter quench at a later time.

\section{CONCLUSIONS}

Using two complementary RG methods, we have provided a comprehensive study of the relaxation dynamics in the Ohmic SBM. The SBM is considered to be the prototype model for a microscopic description of dissipation in open quantum systems. We exploit the mapping of the SBM onto the IRLM. We have studied two basic protocols: a relaxation protocol in which at time $t=0$ the system is prepared in a product state and the time evolution is performed with the SBM Hamiltonian as well as a quench protocol in which in addition at time $t_{q}>0$ a parameter of the Hamiltonian is abruptly changed. While the FRG was used earlier to study quenches in locally correlated quantum systems we here extended the RTRG to this type of nonequilibrium setup. A comparison of the results obtained using our two approximate methods shows that both provide controlled access to the dynamics of the SBM close to the coherent-to-incoherent transition. In the limit of exponentially large times of the relaxation protocol a comparison to established results derived by other approaches is possible and further substantiates this. Crucially, we present new results for intermediate to long times providing a profound understanding of the details of the relaxation dynamics in all time regimes of interest, in particular those relevant for discussing the crossover from coherent to incoherent behavior.

Our first central result concerns the classification of the dynamics in the relaxation protocol with Zeeman splitting $\epsilon=0$ as coherent or incoherent. We have shown that 
for spin-boson couplings $\alpha$ less than $1 / 2$ but close to this value the dynamics should be denoted as partially coherent as only a few local minima (and associated zeros) of the spin expectation value $P(t)$ can be observed on intermediate times while the asymptotic dynamics is purely monotonic. We have confirmed that for $1 / 2<\alpha<1$ the dynamics is monotonic, that is incoherent. The main focus of our work is on the role of the non-Markovian memory in the quench dynamics. We have identified several situations in which the latter dominates the physics. Most prominent are the effects in interaction quenches across the (partially) coherent-to-incoherent transition. In this case nonmonotonic behavior can be transferred deep into a regime in which the time evolution is performed with coupling constant $\alpha>1 / 2$ and $P(t)$ might become monotonic for times at which $\alpha<1 / 2$. Both effects are surprising manifestations of non-Markovian memory. We have shown that the latter plays a subdominant role in the dynamics resulting out of quenches of the Zeeman splitting $\epsilon$.

We finally left the framework of the SBM and considered a quench in a two-lead IRLM with a finite bias voltage applied across the dot. If the level is quenched on resonance with one of the leads, SBM physics is found in this setup. In addition, non-Markovian memory has a sizable effect in this system.

For future research in the field of relaxation and quench dynamics in open quantum systems we note that the RTRG and FRG methods are quite flexible tools to discuss various models, physical quantities and initial setups. Whereas the RTRG requires a weak system-bath coupling, the FRG needs weak local interactions in the quantum system. In this respect the two methods are complementary and a huge parameter regime can be covered. Aside from the local density matrix, other physical observables such as the particle and heat current for biased situations, spectral densities, and explicitly timedependent Hamiltonians can be treated. All correlated initial conditions at time $t=0$ can be studied which can be realized by the time dynamics out of an uncorrelated setup at some past time $t_{0}<0 \underline{\underline{36}}$ Within the RTRG, this means that the system and bath should be decoupled at $t=t_{0}$, whereas for the FRG the total density matrix at $t=t_{0}$ should not contain any correlations from interactions. At $t=0$, correlations are built up by the real-time dynamics such that, in the limit $t_{0} \rightarrow-\infty$, all equilibrium density matrices containing arbitrary local interactions and system-bath correlations can be used as initial condition. Furthermore, by using sequences of different quenches at different times, together with the possibility to choose a finite value for $t_{0}$, many more initial conditions even in nonequilibrium setups can be studied.

An interesting aspect of our approach is the possibility to investigate non-Markovian dynamics of open quantum systems and to analyze the relation to concepts of quantum non-Markovianity discussed within information theoretic approaches $\underline{\underline{5}}$ Several measures have been proposed to characterize the degree to which the dynamics of a given system is non-Markovian. For weak-coupling models our methods provide controlled access to the reduced density matrix, that is all diagonal and nondiagonal matrix elements, from which the proposed measures of nonMarkovianity can be determined. However, for the specific example of the Ohmic SBM close to the coherentto-incoherent transition (at $\alpha=\frac{1}{2}$ ), the coupling constant $\alpha$ is of order 1 and we rely on the mapping to the IRLM, where a weak-coupling expansion in the parameter $g=1-2 \alpha$ is possible. As a consequence, we were only able to discuss the spin expectation value of the SBM in the $z$ direction, since this observable can be directly related to the local occupation probability of the IRLM. In contrast, the spin expectation values in the $x$ or $y$ directions can not be related to the off-diagonal elements of the local density matrix of the IRLM but is a rather complicated nonlinear observable involving reservoir degrees of freedom. Therefore, for the SBM close to $\alpha=\frac{1}{2}$, it would be interesting to investigate the time evolution of the off-diagonal elements of the reduced density matrix using alternative approaches.

\section{ACKNOWLEDGMENT}

We thank U. Weiss for discussions. This work was supported by the DFG via FOR 723 .

\section{Appendix A: Diagrammatics in Liouville space}

We here show how the diagrammatic expansion developed in Ref. 3 for time-translational invariant systems can be extended to the general case of time-dependent Hamiltonians in a straightforward way. We start from a total Hamiltonian of the form $H_{\text {tot }}(t)=H_{\text {res }}(t)+H_{S}(t)+$ $V(t)$, where

$$
\begin{aligned}
H_{\mathrm{res}}(t) & =\sum_{\alpha}\left[H_{\alpha}+\delta \mu_{\alpha}(t) N_{\alpha}\right] \\
& =\sum_{k \nu}\left[\epsilon_{k \nu}+\delta \mu_{\alpha}(t)\right] a_{k \nu}^{\dagger} a_{k \nu}
\end{aligned}
$$

describes a set of noninteracting reservoirs with timedependent chemical potentials $\mu_{\alpha}+\delta \mu_{\alpha}(t)$ ( $\alpha$ denotes the reservoir index and $\nu \equiv \alpha n \sigma$ contains in addition the channel index $n$ and the spin index $\sigma), H_{S}(t)$ is any timedependent Hamiltonian operator describing the isolated local quantum system, and $V(t)$ is a generic interaction between the local system and the reservoirs which, following the notation of Ref. 3 , is written in the compact form $(n=1,2, \ldots)$

$$
V(t)=\frac{1}{n !} g_{12 \ldots n}(t): a_{1} a_{2} \ldots a_{n}:
$$

Here, $a_{1}=\sum_{k} \delta\left(\omega-\epsilon_{k \nu}+\mu_{\alpha}\right)\left(a_{k \nu}^{\dagger} \delta_{\eta+}+a_{k \nu} \delta_{\eta-}\right)$ are the reservoir field operators in continuum notation, and $1 \equiv \eta \nu \omega$ is a multi-index containing the information for 
creation/annihilation operators $(\eta= \pm)$ and characterizing the reservoir state $\left(\epsilon_{k \nu}=\omega+\mu_{\alpha}\right.$ is the energy of the reservoir state). Implicitly we sum over all discrete indices $\eta_{i}$ and $\nu_{i}$ and integrate over the frequencies $\omega_{i}$. : $\cdots$ : denotes normal-ordering w.r.t. the reservoir equilibrium distribution and $g_{12 \ldots n}$ is any vertex operator acting only on the local system characterizing the change of the local state in an interaction process. For its determination for concrete models respecting the correct sequence of fermionic field operators we refer to Ref. 3 .

For times $t<t_{0}$ the local system is assumed to be decoupled from the reservoirs such that the total initial density matrix has the product form

$$
\rho_{\text {tot }}\left(t_{0}\right)=\rho\left(t_{0}\right) \rho_{\text {res }}^{\mathrm{eq}},
$$

where $\rho\left(t_{0}\right)$ is any initial density matrix for the local system and $\rho_{\text {res }}^{\text {eq }}=\prod_{\alpha} \rho_{\alpha}^{\text {eq }}$ describes each reservoir $\alpha$ in grandcanonical equilibrium $\rho_{\alpha}^{\text {eq }}=\frac{1}{Z_{\alpha}} e^{-\left(H_{\alpha}-\mu_{\alpha} N_{\alpha}\right) / T_{\alpha}}$, characterized by its chemical potential $\mu_{\alpha}$ and temperature $T_{\alpha}$ (we set $k_{B}=\hbar=1$ ).

To describe the time evolution for times $t>t_{0}$ we introduce the Liouville operators $L_{\text {tot }}(t)=\left[H_{\text {tot }}(t), \cdot\right]$, $L_{\mathrm{res}}(t)=\left[H_{\mathrm{res}}(t), \cdot\right], L_{S}(t)=\left[H_{S}(t), \cdot\right]$ and $L_{V}(t)=$ $[V(t), \cdot]$, such that the time evolution for the reduced density matrix $\rho(t)=\operatorname{Tr}_{\text {res }} \rho_{\text {tot }}(t)$ of the local system can be written as $(\mathcal{T}$ denotes the time-ordering operator)

$$
\begin{aligned}
\rho(t) & =\operatorname{Tr}_{\mathrm{res}} \mathcal{T} e^{-i \int_{t_{0}}^{t} L_{\mathrm{tot}}\left(t^{\prime}\right) d t^{\prime}} \rho_{\mathrm{tot}}\left(t_{0}\right) \\
& =\left\langle\mathcal{T} e^{-i \int_{t_{0}}^{t}\left[L_{\mathrm{res}}\left(t^{\prime}\right)+L_{S}\left(t^{\prime}\right)+L_{V}\left(t^{\prime}\right)\right] d t^{\prime}}\right\rangle_{\mathrm{res}} \rho\left(t_{0}\right)
\end{aligned}
$$

where $\langle\ldots\rangle_{\text {res }}=\operatorname{Tr}_{\text {res }} \ldots \rho_{\text {res }}^{\text {eq }}$ denotes the average w.r.t. the equilibrium reservoir distribution. Expanding Eq. (A4) in $L_{V}$ we find

$$
\begin{aligned}
\rho(t) & =\Pi\left(t, t_{0}\right) \rho\left(t_{0}\right), \\
\Pi & =\sum_{m=0}^{\infty}(-i)^{m}\left\langle\left(\Pi^{(0)}\left(L_{V} \Pi^{(0)}\right)^{m}\right)\right\rangle_{\mathrm{res}},
\end{aligned}
$$

where $\Pi\left(t, t^{\prime}\right)$ is an effective propagator acting only in Liouville space of the local system, and we used a compact matrix notation in time space with the continuum matrix elements

$$
\begin{aligned}
\Pi^{(0)}\left(t, t^{\prime}\right) & =\theta\left(t-t^{\prime}\right) T e^{-i \int_{t^{\prime}}^{t}\left[L_{\mathrm{res}}(\tau)+L_{S}(\tau)\right] d \tau} \\
L_{V}\left(t, t^{\prime}\right) & =\delta\left(t-t^{\prime}-0^{+}\right) L_{V}(t)
\end{aligned}
$$

To find a diagrammatic representation for $\Pi\left(t, t^{\prime}\right)$ and a self-consistent kinetic equation for $\rho(t)$, we proceed as in Ref. 3 and integrate out the reservoir field operators by using the representation

$$
L_{V}=\frac{1}{n !} \sigma^{p_{1} \ldots p_{n}} G_{1 \ldots n}^{p_{1} \ldots p_{n}}(t): A_{1}^{p_{1}} \ldots A_{n}^{p_{n}}:
$$

where $p_{i}= \pm$ denote the Keldysh indices (over which we sum implicitly), $\sigma^{p_{1} \ldots p_{n}}$ is a Liouvillian sign operator acting on the local system (only necessary for fermions), and $G_{1 \ldots n}^{p_{1} \ldots p_{n}}(t)$ is a Liouvillian vertex operator related to the vertex operator $g_{1 \ldots n}$ (for the precise definitions see Ref. 3). $A_{1}^{p}$ are reservoir field superoperators acting in Liouville space, defined by $A_{1}^{p} b=a_{1} b \delta_{p+}+b a_{1} \delta_{p-}$ when acting on a reservoir operator $b$. Inserting Eq. A9 in Eq. (A6), shifting all field superoperators to the right by using the identity $A_{1}^{p} L_{\mathrm{res}}(t)=\left(L_{\mathrm{res}}(t)-x_{1}(t)\right) A_{1}^{p}$ with $x_{1}(t)=\eta\left(\omega+\mu_{\alpha}+\delta \mu_{\alpha}(t)\right)$, applying Wick's theorem with the following contraction of field superoperators (the upper/lower case refers to bosons/fermions)

$$
\gamma_{11^{\prime}}^{p p^{\prime}}=A_{1}^{p} A_{1^{\prime}}^{p^{\prime}}=\left\{\begin{array}{c}
1 \\
p^{\prime}
\end{array}\right\}\left\langle A_{1}^{p} A_{1^{\prime}}^{p^{\prime}}\right\rangle_{\mathrm{res}}
$$

and using $\operatorname{Tr}_{\text {res }} L_{\text {res }}=0$, we arrive at the diagrammatic representation

$$
\begin{aligned}
\Pi=\Pi_{S} & +\sum_{m=2}^{\infty} \sum_{\text {diagrams }} \frac{( \pm)^{N_{p}}}{S}\left(\prod \gamma\right)(-i)^{m} \\
& \times \Pi_{S}\left(G \Pi_{S}^{X_{1}}\right) \ldots\left(G \Pi_{S}^{X_{m-1}}\right) G \Pi_{S},
\end{aligned}
$$

with

$$
\begin{aligned}
\Pi_{S}^{X}\left(t, t^{\prime}\right) & =\theta\left(t-t^{\prime}\right) T e^{-i \int_{t^{\prime}}^{t}\left(L_{S}+X\right)(\tau) d \tau} \\
G\left(t, t^{\prime}\right) & \equiv\left(G_{1 \ldots n}^{p_{1} \ldots p_{n}}\right)\left(t, t^{\prime}\right) \\
& =\delta\left(t-t^{\prime}-0^{+}\right) G_{1 \ldots n}^{p_{1} \ldots p_{n}}(t)
\end{aligned}
$$

and $\Pi_{S} \equiv \Pi_{S}^{X=0}$. The quantity $X_{i}(t)$ appearing in the propagator $\Pi_{S}^{X_{i}}$ is defined as the sum over all $x_{j}(t)=$ $\eta_{j}\left[\omega_{j}+\mu_{\alpha_{j}}+\delta \mu_{\alpha_{j}}(t)\right]$ from contractions running over this propagator with the index $j$ stemming from the vertex standing left to this propagator. $N_{p}$ is the number of crossings of fermionic contractions and $S=\prod_{i} m_{i}$ ! is a symmetry factor needed for each pair $i$ of vertices connected by $m_{i}$ equivalent lines (see Ref. 3 for more details and the derivation of the diagrammatic rules). $\prod \gamma$ stands for the product of all contractions Eq. A10 and, for brevity, the indices of the vertices $G$ have not been indicated in Eq. A11. Explicitly, the contraction is given by

$$
\gamma_{11^{\prime}}^{p p^{\prime}}=\delta_{1 \overline{1}^{\prime}} p^{\prime}\left\{\begin{array}{l}
\eta \\
1
\end{array}\right\} \rho_{\nu}(\omega) f_{\alpha}\left(p^{\prime} \eta \omega\right)
$$

where $\overline{1} \equiv-\eta, \nu \omega$ and $\delta_{11^{\prime}}=\delta_{\eta \eta^{\prime}} \delta_{\nu \nu^{\prime}} \delta\left(\omega-\omega^{\prime}\right) . \rho_{\nu}(\omega)=$ $\sum_{k} \delta\left(\omega-\epsilon_{k \nu}+\mu_{\alpha}\right)$ denotes the density of states and $f_{\alpha}(\omega)=1 /\left(e^{\omega / T_{\alpha}} \mp 1\right)$ is the Bose/Fermi-function of reservoir $\alpha$.

By grouping all diagrams in sequences of irreducible blocks $\Sigma$ (where each propagator has at least one contraction crossing over it) we find

$$
\Pi=\Pi_{S} \sum_{k=0}^{\infty}\left(-i \Sigma \Pi_{S}\right)^{k},
$$


where

$$
\begin{gathered}
\Sigma=i \sum_{m=2}^{\infty} \sum_{\text {diagrams }} \frac{( \pm)^{N_{p}}}{S}\left(\prod \gamma\right)_{\mathrm{irr}}(-i)^{m} \\
\times G \Pi_{S}^{X_{1}} \ldots G \Pi_{S}^{X_{m-1}} G
\end{gathered}
$$

denotes the sum over all irreducible diagrams indicated by $\left(\prod \gamma\right)_{\text {irr }}$. From (A15) we find that the propagator can be determined from the self-consistent equation

$$
\Pi=\Pi_{S}(1-i \Sigma \Pi) .
$$

Expanding $\Pi_{S}$ in $L_{S}$ we find

$$
\Pi_{S}=\theta\left(1-i L_{S} \Pi_{S}\right),
$$

where $L_{S}\left(t, t^{\prime}\right)=L_{S}(t) \delta\left(t-t^{\prime}-0^{+}\right)$and $\theta\left(t, t^{\prime}\right)=\theta\left(t-t^{\prime}\right)$. Inserting this equation in Eq. (A17) we obtain

$$
\begin{aligned}
\Pi & =\theta(1-i L \Pi) \\
& =\theta \sum_{k=0}^{\infty}(-i L \theta)^{k},
\end{aligned}
$$

where we have introduced the effective Liouvillian

$$
L=L_{S}+\Sigma \text {. }
$$

A self-consistent kinetic equation for $\rho(t)$ can be derived by taking the time derivative of Eqs. A5 and (A19). We find

$$
\partial_{t} \Pi=\delta-i L \Pi
$$

where $\left(\partial_{t} \Pi\right)\left(t, t^{\prime}\right)=\frac{\partial}{\partial t} \Pi\left(t, t^{\prime}\right)$ and $\delta\left(t, t^{\prime}\right)=\delta\left(t-t^{\prime}-0^{+}\right)$. Inserting this relation in $\dot{\rho}(t)=\left(\partial_{t} \Pi\right)\left(t, t_{0}\right) \rho\left(t_{0}\right)$ leads to the kinetic equation

$$
i \dot{\rho}(t)=\int_{t_{0}}^{t} L\left(t, t^{\prime}\right) \rho\left(t^{\prime}\right) d t^{\prime} .
$$

We note that the compact matrix notation in time space allows to write all equations straightforwardly in Fourier space by using the Dirac notation $\langle t \mid E\rangle=$ $\frac{1}{\sqrt{2 \pi}} e^{-i E t}$. This leads to

$$
\begin{aligned}
L_{S}\left(E, E^{\prime}\right) & =\frac{1}{2 \pi} \int e^{i\left(E-E^{\prime}\right) t} L_{S}(t) d t \\
A\left(E, E^{\prime}\right) & =\frac{1}{2 \pi} \iint e^{i E t-i E^{\prime} t^{\prime}} A\left(t, t^{\prime}\right) d t d t^{\prime} \\
\delta\left(E, E^{\prime}\right) & =\frac{1}{2 \pi} \int e^{i\left(E-E^{\prime}\right) t} d t=\delta\left(E-E^{\prime}\right), \\
\theta\left(E, E^{\prime}\right) & =\frac{1}{2 \pi} \iint_{t>t^{\prime}} e^{i E t-i E^{\prime} t^{\prime}} d t d t^{\prime} \\
& =\frac{i}{E} \delta\left(E-E^{\prime}\right),
\end{aligned}
$$

where $A \equiv \Pi, \Pi_{S}, L, \Sigma$. For $\rho(E)$ we use the special definition

$$
\rho(E)=\int d t e^{i E\left(t-t_{0}\right)} \rho(t) \theta\left(t-t_{0}\right),
$$

such that Eqs. (A5) and A23 read in Fourier space

$$
\begin{aligned}
\rho(E) & =\sqrt{2 \pi} e^{-i E t_{0}} \Pi\left(E, t_{0}\right) \rho\left(t_{0}\right), \\
E \rho(E) & =i \rho\left(t_{0}\right) \\
& +\int L\left(E, E^{\prime}\right) e^{-i\left(E-E^{\prime}\right) t_{0}} \rho\left(E^{\prime}\right) d E^{\prime} .
\end{aligned}
$$

For the special case of a time-translational invariant Hamiltonian all objects $A\left(t, t^{\prime}\right)=A\left(t-t^{\prime}\right)$ depend only on the relative time difference and $L_{S}$ is independent of time. This gives

$$
\begin{aligned}
L_{S}\left(E, E^{\prime}\right) & =\delta\left(E-E^{\prime}\right) L_{S} \\
A\left(E, E^{\prime}\right) & =\delta\left(E-E^{\prime}\right) \int_{0}^{\infty} e^{i E t} A(t) d t \\
& \equiv \delta\left(E-E^{\prime}\right) A(E) \\
\Pi\left(E, t_{0}\right) & =\frac{1}{\sqrt{2 \pi}} e^{i E t_{0}} \Pi(E)
\end{aligned}
$$

Inserting Eqs. (A26), A27, A31, and A33 in A18, (A19) and A29 we obtain

$$
\begin{aligned}
\Pi_{S}(E) & =\frac{i}{E-L_{S}}, \\
\Pi(E) & =\frac{i}{E-L(E)}, \\
\rho(E) & =\Pi(E) \rho\left(t_{0}\right) .
\end{aligned}
$$

For the diagrammatic representation of $\Sigma(E)$ we obtain from Eq. A16 the result

$$
\begin{aligned}
& \Sigma(E)=\sum_{m=2}^{\infty} \sum_{\text {diagrams }} \frac{( \pm)^{N_{p}}}{S}\left(\prod \gamma\right)_{\mathrm{irr}} \\
& \times G R_{S}\left(E+X_{1}\right) \ldots G R_{S}\left(E+X_{m-1}\right) G
\end{aligned}
$$

with the resolvent $R_{S}(E)=-i \Pi_{S}(E)=1 /\left(E-L_{S}\right)$. Conveniently, the frequency integration over the variables $\bar{\omega}=\eta \omega$ appearing in the quantities $X_{i}$ are performed by closing the integration contour in the upper half of the complex plane where, except for the density of states and the Bose/Fermi-function appearing in the contraction Eq. (A14), the integrand is analytic.

\section{Appendix B: Quench dynamics}

If, at a certain quench time $t=t_{q}$, the properties of the Hamiltonian are discontinuously changed, it is convenient to define propagators and effective Liouville operators corresponding to the time evolution after and before the quench as well as to the memory by

$$
\begin{aligned}
A_{f}\left(t, t^{\prime}\right) & =A\left(t, t^{\prime}\right) \theta\left(t_{q}-t^{\prime}\right) \\
A_{i}\left(t, t^{\prime}\right) & =\theta\left(t_{q}-t\right) A\left(t, t^{\prime}\right) \\
A_{f i}\left(t, t^{\prime}\right) & =\theta\left(t-t_{q}\right) A\left(t, t^{\prime}\right) \theta\left(t_{q}-t^{\prime}\right),
\end{aligned}
$$


with $A \equiv \Pi, \Pi_{S}, \Sigma, L, L_{S}$. Since $L_{S, f i}=0$ we get $L_{f i}=$ $\Sigma_{f i}$. Due to the form A20 of the propagator we obtain the central equation

$$
\Pi_{f i}\left(t, t^{\prime}\right)=\Pi_{f}\left(t, t_{q}\right) \Pi_{i}\left(t_{q}, t^{\prime}\right)-i\left(\Pi_{f} \Sigma_{f i} \Pi_{i}\right)\left(t, t^{\prime}\right),
$$

which holds in the generic case even if the Hamiltonian is time-dependent before and after the quench. The relation between the density matrix $\rho_{f}(t)=\rho(t) \theta\left(t-t_{q}\right)$ after the quench and the one at the initial time $t_{0}<t_{q}$ before the quench (where the local system and the reservoirs are assumed to be decoupled) can be calculated from

$$
\rho_{f}(t)=\Pi_{f i}\left(t, t_{0}\right) \rho\left(t_{0}\right) .
$$

For the special case of a time-independent Hamiltonian before and after the quench (which we discuss in this paper), the propagators $\Pi_{f / i}$ after and before the quench depend only on the relative time difference. Introducing the special Fourier transformation for the memory parts (where $t_{q}$ is used as reference time and the prefactor $\frac{1}{2 \pi}$ is omitted)

$$
A_{f i}\left(E, E^{\prime}\right)=\iint e^{i E\left(t-t_{q}\right)-i E^{\prime}\left(t^{\prime}-t_{q}\right)} A_{f i}\left(t, t^{\prime}\right) d t d t^{\prime},
$$

and using Eq. (A32) we obtain for Eq. (B4) in Fourier space

$$
\Pi_{f i}\left(E, E^{\prime}\right)=\Pi_{f}(E)\left(1-i \Sigma_{f i}\left(E, E^{\prime}\right)\right) \Pi_{i}\left(E^{\prime}\right),
$$

with

$$
\begin{aligned}
\Pi_{f}(E) & =\frac{i}{E-L_{f}(E)}, \\
\Pi_{i}\left(E^{\prime}\right) & =\frac{i}{E^{\prime}-L_{i}\left(E^{\prime}\right)} .
\end{aligned}
$$

The diagrammatic representation of this formula is illustrated in Fig. 1(a).

The diagrammatic expansion for $-i \Sigma\left(E, E^{\prime}\right)$ can be obtained from Eq. A16 as

$$
\begin{aligned}
& -i \Sigma_{f i}\left(E, E^{\prime}\right)=\iint e^{i E\left(t-t_{q}\right)-i E^{\prime}\left(t^{\prime}-t_{q}\right)} \\
& \times \sum_{m, m^{\prime}=1}^{\infty} \sum_{\text {diagrams }} \frac{( \pm)^{N_{p}}}{S}\left(\prod \gamma\right)_{\mathrm{irr}}(-i)^{m+m^{\prime}} \\
& \times\left(G_{f} \Pi_{S, f}^{X_{1}^{f}} \ldots G_{f} \Pi_{S, f}^{X_{m}^{f}}\right)\left(t, t_{q}\right) \\
& \times\left(\Pi_{S, i}^{X_{m}^{i}} G_{i} \Pi_{S, i}^{X_{1}^{\prime, i}} \ldots G_{i} \Pi_{S, i}^{X_{m^{\prime}-1}} G_{i}\right)\left(t_{q}, t^{\prime}\right) d t d t^{\prime}
\end{aligned}
$$

where $X_{k}^{f}, k=1, \ldots, m$, contain the chemical potentials after the quench, whereas the variables $X_{m}^{i}$ and $X_{k^{\prime}}^{\prime, i}$, $k^{\prime}=1, \ldots, m^{\prime}$ involve the chemical potentials before the quench. Performing the time integrals and using that $G_{f / i}$ is independent of time and $\Pi_{S, f / i}\left(t, t^{\prime}\right)=\Pi_{S, f / i}(t-$ $\left.t^{\prime}\right)$ depends only on the relative time difference, gives a product in Fourier space for the two expressions left and right to the quench with the result

$$
\begin{aligned}
& -i \Sigma_{f i}\left(E, E^{\prime}\right)=\sum_{m, m^{\prime}=1}^{\infty} \sum_{\text {diagrams }} \frac{( \pm)^{N_{p}}}{S}\left(\prod \gamma\right)_{\mathrm{irr}} \\
& \times G_{f} R_{S}^{f}\left(E+X_{1}^{f}\right) \ldots G_{f} R_{S}^{f}\left(E+X_{m}^{f}\right) R_{S}^{i}\left(E^{\prime}+X_{m}^{i}\right) \\
& \times G_{i} R_{S}^{i}\left(E^{\prime}+X_{1}^{\prime, i}\right) \ldots G_{i} R_{S}^{i}\left(E^{\prime}+X_{m^{\prime}-1}^{\prime, i}\right) G_{i}, \quad(\mathrm{~B} 11)
\end{aligned}
$$

with the resolvents $R_{S}^{f / i}(E)=-i \Pi_{S, f / i}(E)=1 /(E-$ $\left.L_{S}^{f / i}\right)$. As a result, we get the usual diagrammatic rules with the difference that the quench time has to be introduced in one propagator, all resolvents left (right) to the quench contain the Fourier variable $E\left(E^{\prime}\right)$, and all vertices, Liouvillians, and chemical potentials appearing left (right) to the quench are associated with the ones after (before) the quench. Furthermore, following Ref. 8, it is possible to sum over all contractions which do not cross over the quench, such that the full effective vertices $G_{f / i}(E)$ and the full resolvents $R_{f / i}(E)=$ $1 /\left[E-L_{f / i}(E)\right]$ appear left/right to the quench with the result

$$
\begin{aligned}
& -i \Sigma_{f i}\left(E, E^{\prime}\right)=\sum_{m, m^{\prime}=1}^{\infty} \sum_{\text {diagrams }} \frac{( \pm)^{N_{p}}}{S}\left(\prod \gamma\right)_{\text {quench }} \\
& \times G_{f}(E) R_{f}\left(E+X_{1}^{f}\right) \ldots G_{f}\left(E+X_{m-1}^{f}\right) R_{f}\left(E+X_{m}^{f}\right) \\
& \times R_{i}\left(E^{\prime}+X_{m}^{i}\right) G_{i}\left(E^{\prime}+X_{m}^{i}\right) R_{i}\left(E^{\prime}+X_{1}^{\prime, i}\right) \ldots \\
& \ldots G_{i}\left(E^{\prime}+X_{1}^{\prime, i}\right) \ldots R_{i}\left(E^{\prime}+X_{m^{\prime}-1}^{\prime, i}\right) G_{i}\left(E^{\prime}+X_{m^{\prime}-1}^{\prime, i}\right) .
\end{aligned}
$$

In this form all contractions have to cross over the quench, indicated by $\left(\prod \gamma\right)_{\text {quench }}$, leading automatically to a connected diagram. Examples of diagrams are shown in Fig. 11(b).

\section{Appendix C: RTRG for IRLM}

Here we present the results of the RTRG method for the IRLM, as they have been derived in Ref. 21 by using the Matsubara cutoff scheme or alternatively in Ref. 31 by the E-RTRG method. We consider the special case of one single reservoir at zero temperature with chemical potential $\mu=0$ and consider the particle-hole symmetric case $\epsilon=0$.

The quantities $Z^{\prime}(E)$ and $\tilde{L}_{\Delta}(E)$ defined in (15) are $4 \times$ 4 matrices in Liouvillian space in the basis $(00,11,10,01)$, where $0 / 1$ denote the unoccupied/occupied state of the local state. Writing each $4 \times 4$ matrix in terms of four $2 \times 2$ blocks, $Z^{\prime}(E)$ and $\tilde{L}_{\Delta}(E)$ can be written as

$$
\begin{aligned}
Z^{\prime}(E) & =\left(\begin{array}{c|c}
\mathbb{1} & 0 \\
\hline 0 & Z(E) \mathbb{1}
\end{array}\right), \\
\tilde{L}_{\Delta}(E) & =-i\left(\begin{array}{c|c}
\Gamma_{1}(E) \tau_{-} & 0 \\
\hline 0 & \frac{1}{2} \Gamma_{2}(E) \mathbb{1}
\end{array}\right),
\end{aligned}
$$


where $\tau_{ \pm}=\frac{1}{2}\left(\mathbb{1} \pm \sigma_{x}\right)$, and the functions $Z(E)=$ $Z\left(-E^{*}\right)^{*}$ and $\Gamma_{1 / 2}(E)=\Gamma_{1 / 2}\left(-E^{*}\right)^{*}$ fulfill the RG equations

$$
\begin{aligned}
\frac{\partial}{\partial E} Z(E) & =U^{2} \frac{Z(E)}{E+i \Gamma_{2}(E) / 2}, \\
\frac{\partial}{\partial E} \Gamma_{1}(E) & =-g \frac{\Gamma_{1}(E)}{E+i \Gamma_{2}(E) / 2}, \\
\frac{\partial}{\partial E} \Gamma_{2}(E) & =-g \frac{\Gamma_{1}(E)}{E+i \Gamma_{1}(E)},
\end{aligned}
$$

with $g=2 U-U^{2}$. The initial conditions at $E=i \omega_{c}$ are given by $Z=1$ and $\Gamma_{1}=\Gamma_{2}=\Gamma_{0}$.

With Eq. (C2), the resolvent $\tilde{R}_{\Delta}(E)=1 /\left(E-\tilde{L}_{\Delta}(E)\right)$ defined in Eq. (14) can be decomposed as

$$
\begin{aligned}
\tilde{R}_{\Delta}(E)=\frac{1}{E} & \left(\begin{array}{c|c}
\tau_{+} & 0 \\
\hline 0 & 0
\end{array}\right)+\frac{1}{E+i \Gamma_{1}(E)}\left(\begin{array}{c|c}
\tau_{-} & 0 \\
\hline 0 & 0
\end{array}\right) \\
& +\frac{1}{E+i \Gamma_{2}(E) / 2}\left(\begin{array}{c|c}
0 & 0 \\
\hline 0 & \mathbb{1}
\end{array}\right)
\end{aligned}
$$

The effective vertices $\tilde{G}_{1}(E)=Z^{\prime}(E) G_{1}(E)$ and $\tilde{G}_{12}(E)=Z^{\prime}(E) G_{12}(E)$, where $G_{1}=\sum_{p} G_{1}^{p}$ and $G_{12}=$ $\sum_{p p^{\prime}} G_{12}^{p p^{\prime}}$ denote the vertices averaged over the Keldysh indices, contain only the index $1 \equiv \eta= \pm$ characterizing creation/annihilation reservoir field operators (note that we consider the single reservoir case without spin). The effective vertices are explicitly given by

$$
\begin{aligned}
& \tilde{G}_{+}(E)=\sqrt{\frac{Z(E) \Gamma_{1}(E)}{2 \pi}} \\
& \times\left(\begin{array}{cc|cc}
2 & & 1 / Z(E) & 0 \\
0 & -1 / Z(E) & 0 \\
\hline 0 & 0 & 0
\end{array}\right), \\
& \tilde{G}_{-}(E)=\sqrt{\frac{Z(E) \Gamma_{1}(E)}{2 \pi}} \\
& \times\left(\begin{array}{ccc}
0 & 0 & -1 / Z(E) \\
0 & 1 / Z(E) \\
\hline 1+i \pi U & 1-i \pi U & 0 \\
0 & 0 &
\end{array}\right), \\
& \tilde{G}_{+-}(E)=-\tilde{G}_{-+}(E)=\left(\begin{array}{c|c}
0 & 0 \\
\hline 0 & U \sigma_{z}
\end{array}\right),
\end{aligned}
$$

and $\tilde{G}_{++}=\tilde{G}_{--}=0$.

\section{Appendix D: Branching point position}

Here we show how to derive the improved formula (77) for the position of the branching point $z_{0}=-i \Gamma_{2}^{*} / 2$ of the resolvent $R_{1}(E)=1 /\left(E+i \Gamma_{1}(E)\right)$, which is at the same time the pole of the resolvent $R_{2}(E)=1 /\left(E+i \Gamma_{2}(E) / 2\right)$, i.e. fulfills the equation $z_{0}=-i \Gamma_{2}\left(z_{0}\right) / 2$ or $\Gamma_{2}\left(z_{0}\right)=\Gamma_{2}^{*}$. We start from the RG equations (35) for $E=z_{0}+i \Lambda$, with $\Lambda>0$, i.e. the regime of the imaginary axis above the branch cut of $R_{1}(E)$. By comparison with the numerical solution we find that a very good approximation consists in replacing $\Gamma_{1 / 2}(E) \rightarrow \Gamma_{2}^{*}$ on the r.h.s. of the RG equations. This leads to the solution Eq. (70) for $\Gamma_{1}(E)$, which, when inserted in the RG equation for $\Gamma_{2}(E)$ and using $E=-i \Gamma_{2}^{*} / 2+i \Lambda$ gives the following differential equation for the determination of $\Gamma_{2}$

$$
\frac{d \Gamma_{2}}{d \Lambda}=-g \frac{T_{K}}{\Lambda+\Gamma_{2}^{*} / 2}\left(\frac{T_{K}}{\Lambda}\right)^{g} .
$$

This equation has to be solved with the boundary conditions

$$
\begin{aligned}
\Gamma_{2} \stackrel{\Lambda \rightarrow \infty}{\longrightarrow} T_{K}\left(\frac{T_{K}}{\Lambda}\right)^{g} \\
\Gamma_{2} \stackrel{\Lambda \rightarrow 0}{\longrightarrow} \Gamma_{2}^{*}
\end{aligned}
$$

Taking another derivative with respect to $\Lambda$ and defining the variable $x=-\Lambda /\left(\Gamma_{2}^{*} / 2\right)$, we obtain the following special case of the hypergeometic differential equation

$$
x(1-x) \frac{d^{2} \Gamma_{2}}{d x^{2}}+(g-(1+g) x) \frac{d \Gamma_{2}}{d x}=0 .
$$

Denoting the hypergeometric function by $F(a, b, c, x)$, this equation has the following two elementary solutions

$$
F(0, g, g, x)=1 \quad(-x)^{1-g} F(1-g, 1,2-g, x) .
$$

Since the hypergeometric function is analytic for $|x|<1$, the second elementary solution has a branch cut from the power law $(-x)^{1-g}$, which is chosen such that the branch cut for $\Gamma_{2}(E)$ lies on the negative imaginary axis. The solution for $|x|<1$ can then be written as

$$
\Gamma_{2}=\Gamma_{2}^{*}+\lambda(-x)^{1-g} F(1-g, 1,2-g, x),
$$

where we have used the boundary condition Eq. (D3) that $\Gamma_{2}=\Gamma_{2}^{*}$ for $x \rightarrow 0$. The coefficient $\lambda$ can be determined by taking the derivative w.r.t. $x$ and comparing with the differential equation (D1) for $x \rightarrow 0$. Using $F(a, b, c, 0)=$ 1 we obtain from Eq. (D6)

$$
\frac{d \Gamma_{2}}{d \Lambda}=-\frac{2}{\Gamma_{2}^{*}} \frac{d \Gamma_{2}}{d x} \stackrel{x \rightarrow 0}{\longrightarrow} \frac{2}{\Gamma_{2}^{*}} \lambda(1-g)\left(\frac{\Gamma_{2}^{*} / 2}{\Lambda}\right)^{g}
$$

which, when compared with Eq. (D1), gives $\lambda=$ $-\frac{g}{1-g} T_{K}\left(\frac{T_{K}}{\Gamma_{2}^{*} / 2}\right)^{g}$, i.e. the solution for $|x|<1$ reads

$$
\begin{aligned}
\Gamma_{2} & =\Gamma_{2}^{*}- \\
& -\frac{g}{1-g} T_{K}\left(\frac{T_{K}}{\Gamma_{2}^{*} / 2}\right)^{g}(-x)^{1-g} F(1-g, 1,2-g, x) .
\end{aligned}
$$

To find the analytical continuation to the regime $|x|>1$ we use the relation 


$$
\begin{aligned}
F(1-g, 1,2-g, x) & =\frac{\Gamma(2-g) \Gamma(g)}{\Gamma(1) \Gamma(1)}(-x)^{g-1} F\left(1-g, 0,1-g, \frac{1}{x}\right)+\frac{\Gamma(2-g) \Gamma(-g)}{\Gamma(1-g) \Gamma(1-g)}(-x)^{-1} F\left(1, g, 1+g, \frac{1}{x}\right) \\
& =(1-g) \frac{\pi}{\sin (\pi g)}(-x)^{g-1}+\frac{1-g}{g} \frac{1}{x} F\left(1, g, 1+g, \frac{1}{x}\right)
\end{aligned}
$$

where we have used $\Gamma(2-g) / \Gamma(1-g)=(1-g), \Gamma(1-g) / \Gamma(-g)=-g$ and $\Gamma(2-g) \Gamma(g)=(1-g) \Gamma(1-g) \Gamma(g)=$ $(1-g) \frac{\pi}{\sin (\pi g)}$ in the last step. Inserting Eq. (D8) in Eq. (D7) gives the following solution for $|x|>1$

$$
\Gamma_{2}=\Gamma_{2}^{*}-\frac{\pi g}{\sin (\pi g)} T_{K}\left(\frac{T_{K}}{\Gamma_{2}^{*} / 2}\right)^{g}+T_{K}\left(\frac{T_{K}}{\Lambda}\right)^{g} F\left(1, g, 1+g, \frac{1}{x}\right) .
$$

Comparing this solution with the asymptotic boundary condition Eq. (D2), we find that the first two terms on the r.h.s. of Eq. (D9) have to cancel each other, leading to the result Eq. (77) for $\Gamma_{2}^{*}$

$$
\frac{\Gamma_{2}^{*}}{2} \approx T_{K}\left(\frac{\pi g}{2 \sin (\pi g)}\right)^{\frac{1}{1+g}} .
$$

\section{Appendix E: Interaction quenches}

In this Appendix we present the detailed calculation of the second term of Eq. 888a for the interaction quench between the coherent and the incoherent regimes. To compute the memory contribution to $P(t)$ we start from Eq. (88b) and restrict ourselves to small couplings $\left|g_{i / f}\right| \ll 1$ such that $g_{i / f} \approx 2 U_{i / f}$. We use the notation $a=i / f, t_{i}=t_{q}$ and $t_{f}=t-t_{q}$, and calculate the functions $F_{\Lambda}^{a}\left(t_{a}\right)$ in leading order in $g_{a}$ by dropping all contributions from the resolvents of order $\mathcal{O}\left(g_{a}\right)$. Therefore, we replace $\Gamma_{2}^{a}(E) \rightarrow \Gamma_{2}^{* a}$ in the resolvent $R_{2}^{a}(E)$ and approximate the $Z$-factors $Z_{a}(E) \approx 1$. The latter follows from the solution of the RG equation (C3), which in leading order reads as

$$
Z_{a}(E) \approx\left(\frac{-i E+\frac{1}{2} \Gamma_{2}^{* a}}{\omega_{c}}\right)^{U_{a}^{2}}
$$

For $|E| \sim T_{K}^{a}$ this gives $Z_{a} \sim\left(T_{K}^{a} / \omega_{c}\right)_{a}^{U_{a}^{2}} \sim 1$ for small $\left|U_{a}\right|$. With these approximations, Eq. (88b) reads as

$$
F_{\Lambda}^{a}\left(t_{a}\right) \approx \frac{1}{2} \sum_{\sigma= \pm} \int \frac{\sqrt{\Gamma_{1}^{a}(E)}}{E+i \Gamma_{1}^{a}(E)} \frac{e^{-i E t_{a}}}{E+i\left(\Lambda+\frac{1}{2} \Gamma_{2}^{* a}\right)-\sigma 0^{+}} \frac{d E}{2 \pi},
$$

where we added a small imaginary part $i \sigma 0^{+}$to $\Lambda$ and have taken the average of $\sigma= \pm$ in order to define the integrand on the branch-cut of the first resolvent. Closing the integration contour in the lower half-plane, there are two pole contributions at $E= \pm \Omega_{a}-i \Gamma_{1}^{* a}$ of the first resolvent (for $g_{a}>0$ ), one pole contribution at $E=-i\left(\Lambda+\frac{1}{2} \Gamma_{2}^{* a}\right)+\sigma 0^{+}$of the second resolvent, and a branch cut contribution starting at $E=-i \frac{1}{2} \Gamma_{2}^{* a}$ from the function $\Gamma_{1}^{a}(E)$. Neglecting terms of $\mathcal{O}\left(g_{a}\right)$, we consider only the contribution of $\mathcal{O}(1)$ of the branch cut. This part is relevant in the regime of intermediate times $\Gamma_{1}^{* a} t_{a} \sim 1 / g_{a}$ and stems from the Lorentzian form of the jump of the resolvent $R_{1}^{a}(E)$ across the branch cut centered at $E \sim-i \Gamma_{1}^{* a}$ [see the discussion around Eq. [80)]. Therefore, we approximate this peak by a true $\delta$-function such that the branch-cut contribution is approximately given by a pole contribution at $E=-i \Gamma_{1}^{* a}$ with $R_{1}^{a}(E) \approx-\operatorname{sign}\left(g_{a}\right) /\left(E+i \Gamma_{1}^{* a}\right)$ close to this pole according to Eq. (80).

Using Eq. (70) for $\Gamma_{1}^{a}(E)$ and neglecting terms $\mathcal{O}\left(g_{a}\right)$, we can split the various pole contributions as

$$
F_{\Lambda}^{a}\left(t_{a}\right) \approx F_{\Lambda, 1}^{a}\left(t_{a}\right)+F_{\Lambda, 2}^{a}\left(t_{a}\right),
$$

with

$$
\begin{gathered}
F_{\Lambda, 1}^{a}\left(t_{a}\right)=\left(2 \cos \left(\Omega_{a} t_{a}\right) \theta\left(g_{a}\right)-\operatorname{sign}\left(g_{a}\right)\right) e^{-\Gamma_{1}^{* a} t_{a}} \\
\times \operatorname{Re} \frac{\sqrt{\Gamma_{1}^{* a}}}{\Gamma_{1}^{* a}-\frac{1}{2} \Gamma_{2}^{* a}-\Lambda+i 0^{+}}, \\
F_{\Lambda, 2}^{a}\left(t_{a}\right)=-\operatorname{Re} \frac{\sqrt{T_{K}^{a}}\left[\frac{T_{K}^{a}}{\Lambda}\right]^{g_{a} / 2} e^{-\left(\Lambda+\frac{1}{2} \Gamma_{2}^{* a}\right) t_{a}}}{T_{K}^{a}\left[\frac{T_{K}^{a}}{\Lambda}\right]^{g_{a}}-\frac{1}{2} \Gamma_{2}^{* a}-\Lambda+i 0^{+}} .
\end{gathered}
$$

Note that when considering the product $F_{\Lambda}^{f}\left(t_{f}\right) F_{\Lambda}^{i}\left(t_{i}\right)$ entering the second term for $P(t)$ in Eq. 888a), there is no divergence for the subsequent integral over $\Lambda$ close to $\Lambda \sim \Gamma_{1}^{* a}-\frac{1}{2} \Gamma_{2}^{* a}$ since the principal values of the two resolvents are centered at different positions. The combination $F_{\Lambda, 1}^{f}\left(t_{f}\right) F_{\Lambda, 1}^{i}\left(t_{i}\right)$ will be neglected in the following since it has the strongest decay $\sim e^{-\Gamma_{1}^{* f} t_{f}} e^{-\Gamma_{1}^{* i} t_{i}}$. For the case $g_{a}=-g_{\bar{a}}<0$ (with $\bar{a}=f / i$ for $a=i / f$ ), the combination $F_{\Lambda, 2}^{a}\left(t_{a}\right) F_{\Lambda, 1}^{\bar{a}}\left(t_{\bar{a}}\right)$ contains an exponentially decaying factor $e^{-\Lambda t_{a}}$ for the integration over $\Lambda$ in 88ad. This restricts the integration range to $\Lambda \lesssim 1 / t_{a} \ll \Gamma_{1}^{* a}<\Gamma_{1}^{*, \bar{a}}$, where we have used that $\Gamma_{1}^{* a} \approx 2^{g_{a}} T_{K}^{a}$ is larger for a positive interaction than for a negative one. Therefore, we can neglect $-\Lambda+i 0^{+}$in all denominators of the resolvents occurring in Eqs. (E4) and (E5). Furthermore, we can approximate $\left(T_{K}^{a} / \Lambda\right)^{g_{a}} \approx\left(T_{K}^{a} t_{a}\right)^{g_{a}}$ in Eq. (E5). The 
same holds for the combination $F_{\Lambda, 2}^{f}\left(t_{f}\right) F_{\Lambda, 2}^{i}\left(t_{i}\right)$, except that an exponential factor $e^{-\Lambda\left(t_{i}+t_{f}\right)}=e^{-\Lambda t}$ occurs such that we get $\left(T_{K}^{a} / \Lambda\right)^{g_{a}} \approx\left(T_{K}^{a} t\right)^{g_{a}}$ in Eq. (E5). With these approximations we can easily calculate the final integral over $\Lambda$ to get the second term of Eq. (88a) for the two quench protocols.

a. In the coherent to incoherent quench the coupling before $t_{q}$ is positive and has the same absolute value as the one afterwards: $g_{i}=-g_{f}=g>0$. The combinations $F_{\Lambda, 2}^{f}\left(t_{f}\right) F_{\Lambda, 1}^{i}\left(t_{i}\right)$ and $F_{\Lambda, 2}^{f}\left(t_{f}\right) F_{\Lambda, 2}^{i}\left(t_{i}\right)$ are both purely decaying after the quench with decay rate $\frac{1}{2} \Gamma_{2}^{* f}$. However, since $F_{\Lambda, 1}^{i}\left(t_{i}\right) \sim e^{-\Gamma_{1}^{* i} t_{i}}$ and $F_{\Lambda, 2}^{i}\left(t_{i}\right) \sim e^{-\frac{1}{2} \Gamma_{2}^{* i} t_{i}}$, the combination $F_{\Lambda, 2}^{f}\left(t_{f}\right) F_{\Lambda, 2}^{i}\left(t_{i}\right)$ will dominate. Together with $-g_{i}=-g, \Gamma_{2}^{* i} \approx 2^{g} T_{K}^{i}$ and $\Gamma_{2}^{* f} \approx 2^{-g} T_{K}^{f}$, this leads to the result

$$
\begin{gathered}
-g_{i} \int_{0}^{\infty} F_{\Lambda, 2}^{f}\left(t-t_{q}\right) F_{\Lambda, 2}^{i}\left(t_{q}\right) d \Lambda \approx-g \frac{e^{-\frac{1}{2} \Gamma_{2}^{* i} t_{q}-\frac{1}{2} \Gamma_{2}^{* f}\left(t-t_{q}\right)}}{t} \\
\quad \times \frac{1}{\sqrt{T_{K}^{i} T_{K}^{f}}} \frac{\left(T_{K}^{i} t / 2\right)^{g / 2}}{\left(T_{K}^{i} t / 2\right)^{g}-\frac{1}{2}} \frac{\left(T_{K}^{f} t / 2\right)^{-g / 2}}{\left(T_{K}^{f} t / 2\right)^{-g}-\frac{1}{2}} . \quad \text { (E6) }
\end{gathered}
$$

Introducing the function $S_{x}^{ \pm}$defined in Eq. (90) in the main text, we obtain the second term of Eq. (89).

$b$. The incoherent to coherent quench has the opposite signs of the couplings $g_{f}=-g_{i}=g>0$. Here we consider both combinations $F_{\Lambda, 1}^{f}\left(t_{f}\right) F_{\Lambda, 2}^{i}\left(t_{i}\right)$ and $F_{\Lambda, 2}^{f}\left(t_{f}\right) F_{\Lambda, 2}^{i}\left(t_{i}\right)$ since the first one is oscillating after the quench whereas the second one is purely decaying. Using $\Gamma_{1 / 2}^{* i} \approx 2^{-g} T_{K}^{i}$ and $\Gamma_{1 / 2}^{* f} \approx 2^{g} T_{K}^{f}$, we obtain

$$
\begin{aligned}
& \int_{0}^{\infty} F_{\Lambda, 1}^{f}\left(t-t_{q}\right) F_{\Lambda, 2}^{i}\left(t_{q}\right) d \Lambda \approx-\left(2 \cos \left(\Omega_{f}\left(t-t_{q}\right)\right)-1\right) \\
& \times \frac{e^{-\frac{1}{2} \Gamma_{2}^{* i} t_{q}-\Gamma_{1}^{* f}\left(t-t_{q}\right)}}{t_{q}} \frac{2}{\sqrt{T_{K}^{* i} T_{K}^{* f}}} \frac{\left(T_{K}^{i} t_{q} / 2\right)^{-g / 2}}{\left(T_{K}^{i} t_{q} / 2\right)^{-g}-\frac{1}{2}},
\end{aligned}
$$

and

$$
\begin{gathered}
\int_{0}^{\infty} F_{\Lambda, 2}^{f}\left(t-t_{q}\right) F_{\Lambda, 2}^{i}\left(t_{q}\right) d \Lambda \approx \frac{e^{-\frac{1}{2} \Gamma_{2}^{* i} t_{q}-\frac{1}{2} \Gamma_{2}^{* f}\left(t-t_{q}\right)}}{t} \\
\times \frac{1}{\sqrt{T_{K}^{* i} T_{K}^{* f}}} \frac{\left(T_{K}^{i} t / 2\right)^{-g / 2}}{\left(T_{K}^{i} t / 2\right)^{-g}-\frac{1}{2}} \frac{\left(T_{K}^{f} t / 2\right)^{g / 2}}{\left(T_{K}^{f} t / 2\right)^{g}-\frac{1}{2}} .
\end{gathered}
$$

For $t-t_{q} \ll t_{q}$, we can replace $t$ by $t_{q}$ in the last equation. Furthermore, using Eq. (86), we can use for $T_{K}^{i} t_{q} \gg 1$

$$
P^{i}\left(t_{q}\right) \approx g e^{-\frac{1}{2} \Gamma_{2}^{* i} t_{q}} \frac{\left(S_{T_{K}^{i} t_{q}}^{-}\right)^{2}}{T_{K}^{i} t_{q}}
$$

Therefore, the sum of the two contributions (E7) and (E8) multiplied by $-g_{i}=g$ (i.e. the contribution to $P(t)$ from the quench) can be written as

$$
\begin{gathered}
-g_{i} \int_{0}^{\infty} F_{\Lambda}^{f}\left(t-t_{q}\right) F_{\Lambda, 2}^{i}\left(t_{q}\right) d \Lambda \approx \\
\approx A\left[-\frac{2}{S_{T_{K}^{i} t_{q}}^{-}} e^{-\Gamma_{1}^{* f}\left(t-t_{q}\right)}\left(2 \cos \left(\Omega_{f}\left(t-t_{q}\right)\right)-1\right)+\right. \\
\left.+\frac{S_{T_{K}^{f} t_{q}}^{+}}{S_{T_{K}^{i} t_{q}}^{-}} e^{-\Gamma_{2}^{* f}\left(t-t_{q}\right) / 2}\right] P^{i}\left(t_{q}\right), \quad(\mathrm{E} 10)
\end{gathered}
$$

where $A=\sqrt{T_{K}^{i} / T_{K}^{f}}=\left(\Gamma_{0} / \omega_{c}\right)^{g}<1$. This leads straightforwardly to Eq. (91).
1 A. J. Leggett, S. Chakravarty, A. T. Dorsey, M. P. A. Fisher, A. Garg, and W. Zwerger, Rev. Mod. Phys. 59 (1), 1 (1987).

2 U. Weiss, Quantum dissipative system (World Scientific, Singapore, 1999).

${ }^{3}$ H. Schoeller, Eur. Phys. J. Special Topics 168, 179 (2009).

4 W. Metzner, M. Salmhofer, C. Honerkamp, V. Meden, and K. Schönhammer, Rev. Mod. Phys. 84, 299 (2012).

5 H.P. Breuer, J. Phys. B 45, 154001 (2012).

6 M. Pletyukhov, D. Schuricht, and H. Schoeller, Phys. Rev. Lett. 104, 106801 (2010).

7 O. Kashuba and H. Schoeller, Phys. Rev. B 87, 201402 (2013).

8 M. Pletyukhov and H. Schoeller, Phys. Rev. Lett. 108, 260601 (2012).
9 A. A. Slutskin, K. N. Bratus, A. Bergvall, and V. S. Shumeiko, Europhys. Lett. 96, 40003 (2011).

10 R. Egger, H. Grabert, and U. Weiss, Phys. Rev. E 55(4), R3809 (1997).

11 D. M. Kennes, O. Kashuba, M. Pletyukhov, H. Schoeller, and V. Meden, Phys. Rev. Lett. 110, 100405 (2013).

12 The prefactor of $P_{\text {pole }}(t)$ agrees with the one of NIBA only to order $g^{0}$. We were informed by U. Weiss that computing it within improved NIBA $^{10}$ gives a result which agrees to ours to order $g$.

13 D. P. DiVincenzo and D. Loss, Phys. Rev. B 71, 035318 (2005).

14 R. Egger and C. H. Mak, Phys. Rev. B 50, 15210 (1994).

15 F. B. Anders and A. Schiller, Phys. Rev. B 74, 245113 (2006). 
16 H. Wang and M. Thoss, New J. Phys. 10, 115005 (2008).

17 P. P. Orth, A. Imambekov, and K. Le Hur, Phys. Rev. A 82, 032118 (2010).

18 P. P. Orth, A. Imambekov, and K. Le Hur, Phys. Rev. B 87, 014305 (2013).

19 D. R. Reichman, F. L. H. Brown, and P. Neu, Phys. Rev. E 55, 2328 (1997).

20 H. Schoeller and F. Reininghaus, Phys. Rev. B 80, 045117 (2009); ibid. Phys. Rev. B 80, 209901(E) (2009).

21 S. Andergassen, M. Pletyukhov, D. Schuricht, H. Schoeller, and L. Borda, Phys. Rev. B 83, 205103 (2011).

${ }^{22}$ D. M. Kennes, S. G. Jakobs, C. Karrasch, and V. Meden, Phys. Rev. B 85, 085113 (2012).

23 D. M. Kennes and V. Meden, Phys. Rev. B 87, 075130 (2013).

24 M. Grifoni and P. Hänggi, Physics Reports 304(5-6), 229 (1998).

25 M. Keil and H. Schoeller, Phys. Rev. B 63, 180302 (2001).

26 A. Hackl and S. Kehrein, Phys. Rev. B 78, 092303 (2008).

27 A. Alvermann and H. Fehske, Phys. Rev. Lett. 102, 150601 (2009).

28 P. P. Orth, D. Roosen, W. Hofstetter, and K. Le Hur, Phys. Rev. B 82, 144423 (2010).

29 D. Kast and J. Ankerhold, Phys. Rev. Lett. 110, 010402 (2013).

30 D. Kast and J. Ankerhold, arXiv:1301.1772 (2013).
31 H. Schoeller, in preparation.

32 R. Gezzi, T. Pruschke, and V. Meden, Phys. Rev. B 75, 045324 (2007).

33 S. G. Jakobs, V. Meden, and H. Schoeller, Phys. Rev. Lett. 99, 150603 (2007).

34 S. G. Jakobs, M. Pletyukhov, and H. Schoeller, Phys. Rev. B 81, 195109 (2010).

35 C. Karrasch, S. Andergassen, M. Pletyukhov, D. Schuricht, L. Borda, V. Meden, and H. Schoeller, Europhys. Lett. 90, 30003 (2010).

36 D. M. Kennes and V. Meden, Phys. Rev. B 85, 245101 (2012).

37 H. Haug and A.-P. Jauho, Quantum kinetics in transport and optics of semiconductors (Springer Verlag, Berlin, 2008).

38 J. Rammer, Quantum Field Theory of Non-equilibrium States (Cambridge University Press, Cambridge, 2007).

39 L. Borda, K. Vladár, and A. Zawadowski, Phys. Rev. B 75, 125107 (2007).

40 L. Borda, A. Schiller, and A. Zawadowski, Phys. Rev. B 78, 201301 (2008).

41 F. Lesage and H. Saleur, Phys. Rev. Lett. 80, 4370 (1998).

42 E. Eidelstein, A. Schiller, F. Güttge, and F. B. Anders, Phys. Rev. B 85, 075118 (2012).

${ }^{43}$ F. Güttge, F. B. Anders, U. Schollwöck, E. Eidelstein, and A. Schiller, Phys. Rev. B 87, 115115 (2013). 